\title{
COVID-19 Variant Detection with a High-Fidelity CRISPR-Cas12 Enzyme
}

Clare L. Fasching ${ }^{1 *}$, Venice Servellita ${ }^{2,3 \star}$, Bridget McKay ${ }^{1}$, Vaishnavi Nagesh ${ }^{1}$, James

P. Broughton ${ }^{1}$, Alicia Sotomayor-Gonzalez ${ }^{2,3}$, Baolin Wang ${ }^{2,3}$, Noah Brazer ${ }^{2,3}$, Kevin Reyes $^{2,3}$, Jessica Streithorst ${ }^{2,3}$, Rachel N. Deraney ${ }^{1}$, Emma Stanfield ${ }^{1}$, Carley G.

Hendriks ${ }^{1}$, Steve Miller ${ }^{2,3}$, Jesus Ching ${ }^{1}$, Janice S. Chen ${ }^{1 \dagger}$, Charles Y. Chiu ${ }^{2,3,4 \dagger}$

${ }^{1}$ Mammoth Biosciences, Inc., Brisbane, CA, USA.

${ }^{2}$ Department of Laboratory Medicine, University of California San Francisco, San Francisco, CA, USA.

${ }^{3}$ UCSF-Abbott Viral Diagnostics and Discovery Center, San Francisco, CA, USA.

${ }^{4}$ Department of Medicine, Division of Infectious Diseases, University of California San Francisco, San Francisco, CA, USA.

*These authors contributed equally

${ }^{\dagger}$ Co-corresponding authors

\section{Abstract}

Laboratory tests for the accurate and rapid identification of SARS-CoV-2 variants can potentially guide the treatment of COVID-19 patients and inform infection control and public health surveillance efforts. Here we present the development and validation of a rapid COVID-19 variant DETECTR ${ }^{\circledR}$ assay incorporating loop-mediated isothermal amplification (LAMP) followed by CRISPR-Cas 12 based identification of single nucleotide polymorphism (SNP) mutations in the SARS-CoV-2 spike (S) gene. This assay targets the L452R, E484K/Q/A, and N501Y mutations that are associated with 
nearly all circulating viral lineages and identifies the two circulating variants of concern, Delta and Omicron. In a comparison of three different Cas12 enzymes, only the newly identified enzyme CasDx1 was able to accurately identify all targeted SNP mutations. An analysis pipeline for CRISPR-based SNP identification from 139 clinical samples yielded an overall SNP concordance of $98 \%$ and agreement with SARS-CoV-2 lineage classification of 138/139 compared to viral whole-genome sequencing. We also showed that detection of the single E484A mutation was necessary and sufficient to accurately identify Omicron from other major circulating variants in patient samples. These findings demonstrate the utility of CRISPR-based DETECTR ${ }^{\circledR}$ as a faster and simpler diagnostic than sequencing for SARS-CoV-2 variant identification in clinical and public health laboratories.

\section{Introduction}

The emergence of new SARS-CoV-2 variants threatens to substantially prolong the COVID-19 pandemic. SARS-CoV-2 variants, especially Variants of Concern (VOCs) ${ }^{1,2}$, have caused resurgent COVID-19 outbreaks in the United States $^{2-5}$ and worldwide ${ }^{1,6,7}$, even in populations with a high proportion of vaccinated individuals ${ }^{8-11}$. Mutations in the spike protein, which binds to the human ACE2 receptor, can render the virus more infectious and/or more resistant to antibody neutralization, resulting in increased transmissibility ${ }^{12}$, and/or escape from immunity, whether vaccine-mediated or naturally acquired immunity ${ }^{13,14}$. Variant identification can also be clinically significant, as some 
mutations substantially reduce the effectiveness of available monoclonal antibody therapies for the disease ${ }^{15}$.

Tracking the evolution and spread of SARS-CoV-2 variants in the community can inform public policy regarding testing and vaccination, as well as guide contact tracing and containment effects during local outbreaks ${ }^{16,17}$. Virus whole-genome sequencing (WGS) and single nucleotide polymorphism (SNP) genotyping are commonly used to identify variants $^{16,18}$, but can be limited by long turnaround times and/or the requirement for bulky and expensive laboratory instrumentation. Diagnostic assays based on clustered interspaced short palindromic repeats (CRISPR $)^{19}$ have been developed for rapid detection of SARS-CoV-2 in clinical samples ${ }^{13,20-23}$, and a few have obtained Emergency Use Authorization (EUA) by the US Food and Drug Administration (FDA) ${ }^{24-}$ ${ }^{26}$. Some advantages of these assays for use in laboratory and point of care settings include low cost, minimal instrumentation, and a sample-to-answer turnaround time of under 2 hours ${ }^{20,23,27-29}$.

Here we present the development of a CRISPR-based COVID-19 variant DETECTR ${ }^{\circledR}$ assay (henceforth abbreviated as DETECTR ${ }^{\circledR}$ assay) for the detection of SARS-CoV-2 mutations and evaluate its performance on a total of 139 patient respiratory swab samples using WGS as a comparator method (Fig. 1a). The assay combines RT-LAMP pre-amplification followed by fluorescent detection using a CRISPR-Cas12 enzyme. We perform a comparative evaluation of multiple candidate Cas 12 enzymes and demonstrate that robust assay performance depends on the specificity of the newly 
identified CRISPR-Cas12 enzyme called CasDx1 in identifying key SNP mutations of functional relevance in the spike protein at amino acid positions 452,484 and $501^{30}$.

\section{Results}

\section{Identifying the optimal CRISPR-Cas12 enzyme for SNP detection}

To determine the optimal Cas12 enzyme for SNP detection, we evaluated three different CRISPR-Cas effectors with trans-cutting activity: LbCas12a, AsCas12a, and a novel Cas12 enzyme called CasDx1. We initially screened guide RNAs (gRNAs) with CasDx1 and LbCas12a for activity on synthetic gene fragments encoding regions of the SARS-CoV-2 S-gene with either wild-type (WT) or mutant (MUT) sequences at amino acid positions 452, 484, and 501 (Fig. 1b-c). From this initial activity screen, we identified the top-performing gRNAs for each S-gene variant encoding either L452R, E484K or N501Y (Fig. 1d). Further evaluation of these guides using CasDx1, LbCas12a and AsCas12a with their cognate gRNAs on synthetic gene fragments revealed differences in SNP differentiation capabilities, with CasDx1 showing the clearest SNP differentiation between wild-type (WT) and mutant (MUT) sequences for all targeted Sgene variants (Fig. 1d and Extended Data Fig. 1a). In comparison, LbCas12a could differentiate SNPs at positions 452 and 484, but not 501, whereas AsCas12a could only differentiate the SNP at position 452 (Fig. 1d and Extended Data Fig. 1a).

We next tested SNP differentiation capabilities on heat-inactivated viral cultures using the full DETECTR ${ }^{\circledR}$ assay, consisting of RNA extraction, multiplexed RT-LAMP amplification (Fig. 1C), and CRISPR-Cas12 detection with guide RNAs targeting part of 
the spike receptor-binding domain (RBD) (Fig. 1b). The LAMP primer design incorporated two sets of six primers each, with both sets generating overlapping spike RBD amplicons that spanned the L452R, E484K, and N501Y mutations. We chose to adopt a redundant LAMP design for two reasons: first, this approach was shown to improve detection sensitivity in initial experiments; second, we sought to increase assay robustness given the continual emergence of escape mutations in the spike RBD throughout the course of the pandemic ${ }^{13}$. The tested viral cultures included an ancestral SARS-CoV-2 lineage (WA-1) containing the wild-type spike protein (D614) targeted by the approved mRNA (BNT162b2 from Pfizer or mRNA-1273 from Moderna) ${ }^{31,32}$ and DNA adenovirus vector (Ad26.COV2.S from Johnson and Johnson) ${ }^{33}$ vaccines, variants being monitored (VBMs) that were previously classified as VOCs or variants of interest (VOls), including Alpha (B.1.1.7), Beta (B.1.351), Gamma (P.1), Epsilon (B.1.427 and B.1.429), Kappa (B.1.617.1), and Zeta (P.2) lineages, and the current VOC Delta (B.1.617.2) lineage ${ }^{34}$. Heat-inactivated viral culture samples representing the seven SARS-CoV-2 lineages were quantified by digital droplet PCR across a 4-log dynamic range and used to evaluate the analytical sensitivity of the pre-amplification step. RTLAMP amplification was evaluated using six replicates from each viral culture. We observed consistent amplification for all seven SARS-CoV-2 lineages with 10,000 copies of target input per reaction $(200,000$ copies $/ \mathrm{mL})$ (Fig. 1e), which is comparable to the target input of $>200,000$ copies $/ \mathrm{mL}$ viruses ( $<30 \mathrm{Ct}$ value) required for sequencing workflows used in SARS-CoV-2 variant surveillance ${ }^{35,36}$. 
To evaluate the specificity of the different Cas12 enzymes, amplified material from each viral culture was pooled and the SNPs resulting in the L452R, E484K and N501Y mutations were detected using CasDx1, LbCa12a and AsCas12a. Similar to the results found using gene fragments, CasDx1 correctly identified the wild-type (WT) and mutational (MUT) targets at positions 452, 484 and 501 in each LAMP-amplified, heatinactivated viral culture (Fig. 1 and Extended Data Fig. 1b). In comparison, LbCas12a could differentiate WT from MUT at position 501 on LAMP-amplified viral cultures but showed much higher background for the WT target at position 452 and higher background for both WT and MUT targets at position 484 for (Fig. 1f and Extended Data Fig. 1b). Additionally, AsCas12a could differentiate WT from MUT targets at position 452 albeit with substantial background but was unable to differentiate WT from MUT targets at positions 484 and 501 (Fig. 1f and Extended Data Fig. 1b). From these data, we concluded that CasDx1 would provide more consistent and accurate calls for the L452R, E484K and N501Y mutations. We thus proceeded to further develop the assay using only the high-fidelity CasDx1 enzyme. 
medRxiv preprint doi: https://doi.org/10.1101/2021.11.29.21267041; this version posted February 3, 2022. The copyright holder for this preprint (which was not certified by peer review) is the author/funder, who has granted medRxiv a license to display the preprint in perpetuity.

a

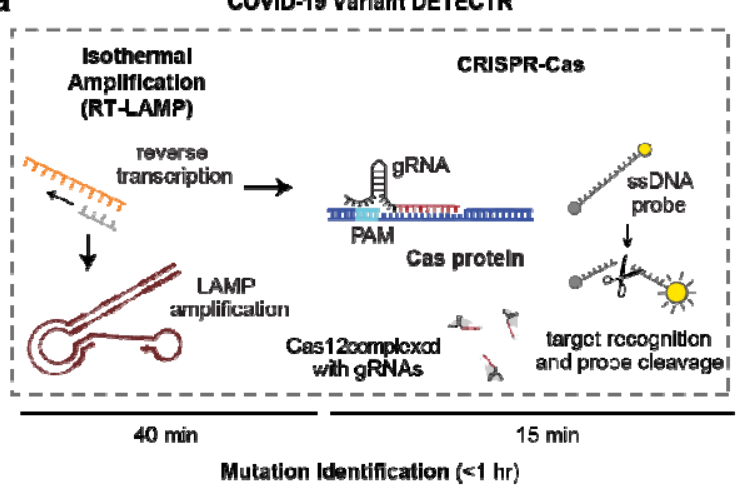

SARS-CoV-2 Whole Genome Sequencing

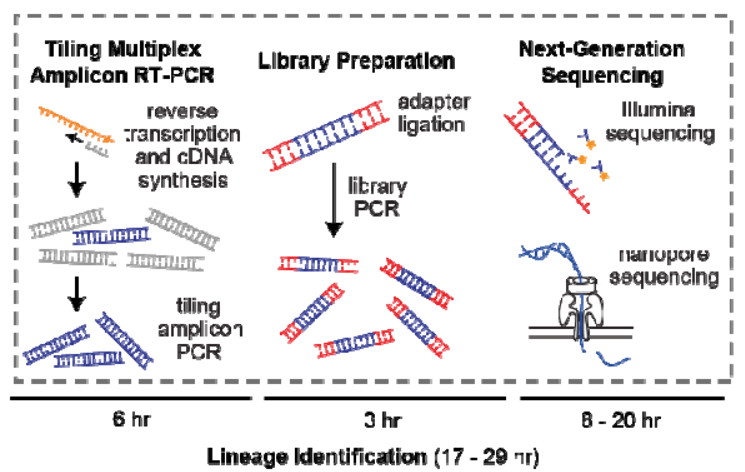

b

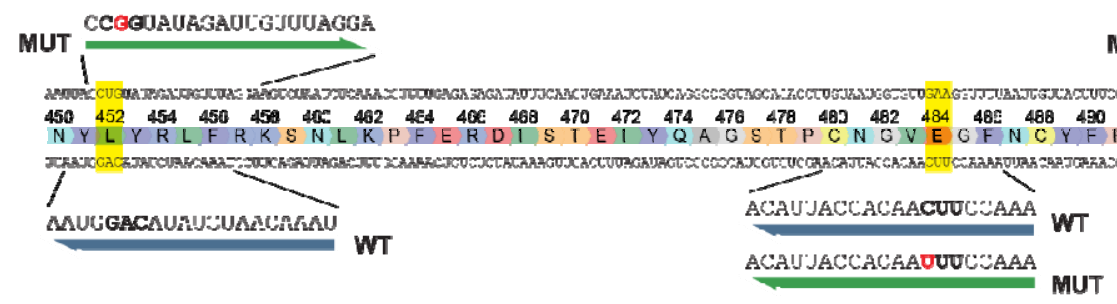

C

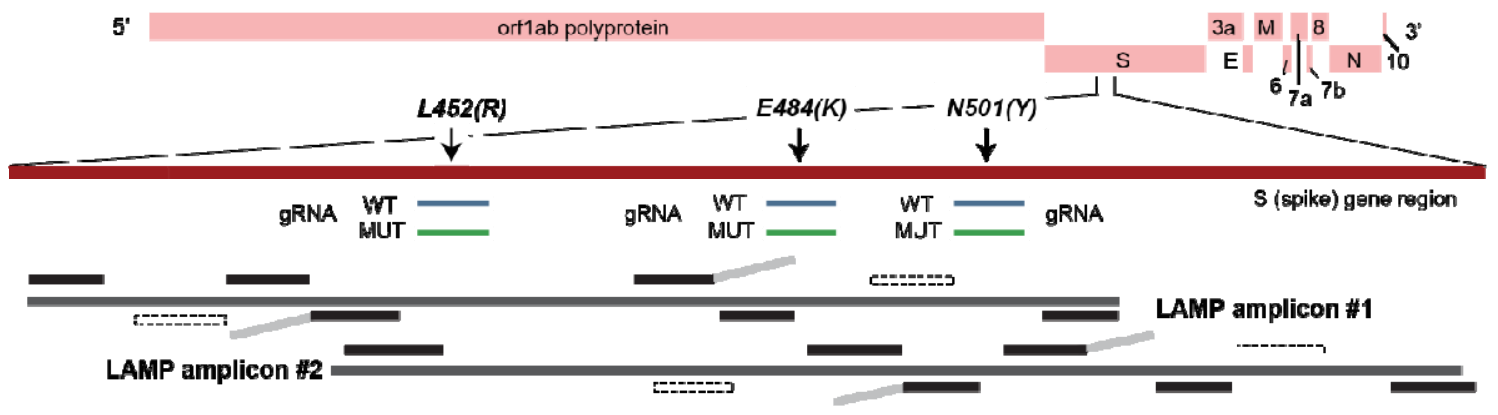

d

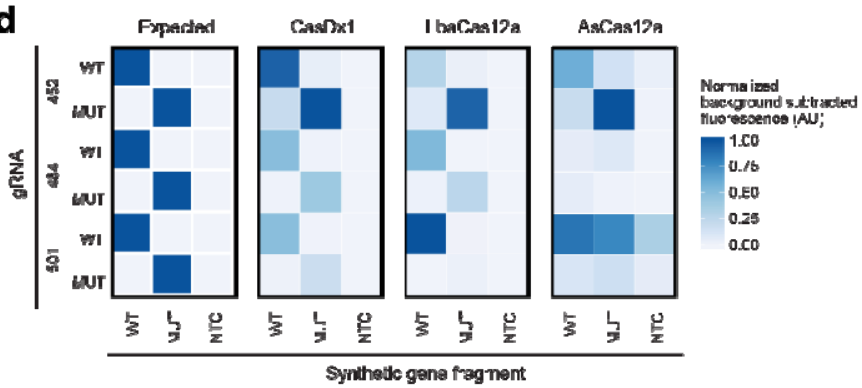

WT CA.ACCCACUAAUCGJGUUGG

MUT CAACCCACUUAUGG:IGUU⿴囗十⺝

$\theta$

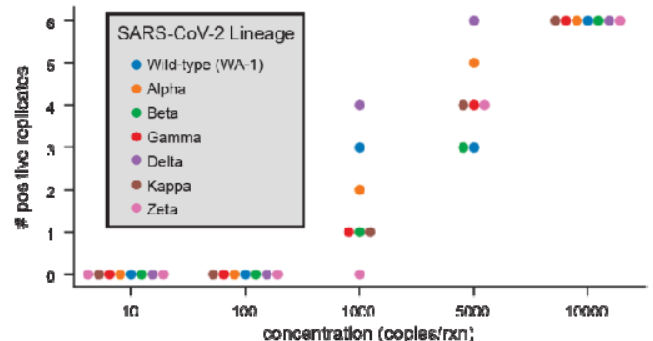

f
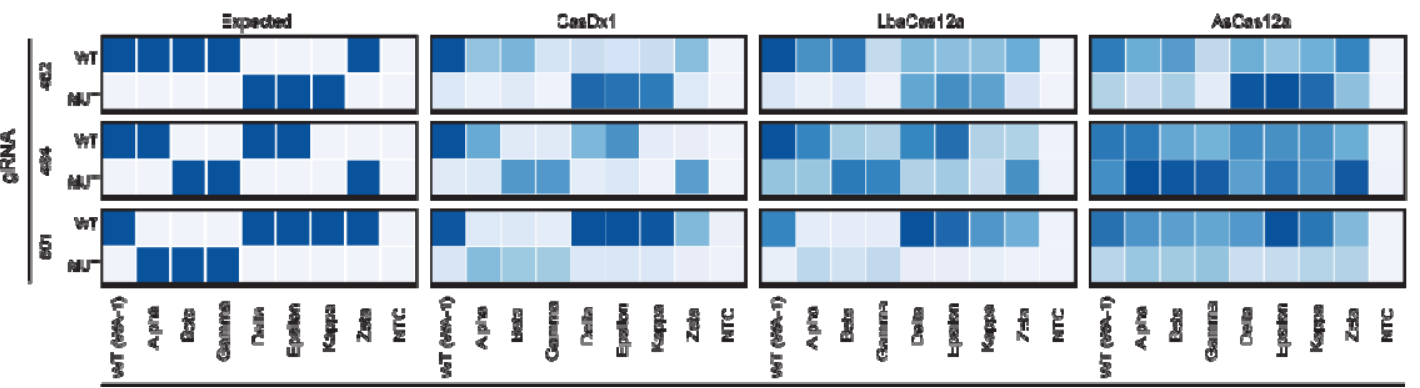

Aremellaw

1.00

a.s

0.60

0.00

toatinactivated Viral Cutures 
Fig. 1 | Design and Workflow for the DETECTR ${ }^{\circledR}$ assay. a, Workflow comparison between the DETECTR ${ }^{\circledR}$ assay and SARS-CoV-2 whole-genome sequencing (WGS). b, Schematic of CRISPR-Cas gRNA design for SARS-CoV-2 S gene mutations. c, Schematic of multiplexed RT-LAMP primer design showing the SARS-CoV-2 S gene mutations and gRNA positions. d, Heat map comparison of three different Cas12 enzymes tested using $10 \mathrm{nM}$ PCR-amplified synthetic gene fragments $(t=30$ minutes). e, Dot plot showing the number $(n=6)$ of positive replicates across a 4-log dynamic range of the RT-LAMP products. $\mathbf{f}$, Heat map comparison of end-point fluorescence $(t=$ 30 minutes) of three different Cas12 enzymes tested against heat-inactivated viral cultures. Replicates $(n=6)$ generated using RT-LAMP were pooled and CRISPR-Cas12 reactions were then run in triplicate $(n=3)$.

\section{Data analysis pipeline for calling COVID-19 variant SNPs with the DETECTR ${ }^{\circledR}$}

\section{assay}

To develop a data analysis pipeline for calling SARS-CoV-2 SNP mutations and assign lineage classifications with the DETECTR ${ }^{\circledR}$ assay (Fig. 2a-b), we first used data collected from SNP synthetic gene fragment controls $(n=279)$ that included all mutational combinations of 452, 484 and 501 (see Methods). Based on the control sample data, we generated allele discrimination plots ${ }^{37,38}$ to define boundaries that separated the WT and MUT signals (Extended Data Fig. 4a). Clear differentiation between WT and MUT signals was observed when plotting the ratio against the average of the WT and MUT transformed values on a mean average (MA) plot ${ }^{37,38}$ (Extended 
medRxiv preprint doi: https://doi.org/10.1101/2021.11.29.21267041; this version posted February 3, 2022. The copyright holder for this preprint (which was not certified by peer review) is the author/funder, who has granted medRxiv a license to display the preprint in perpetuity.

It is made available under a CC-BY-NC-ND 4.0 International license .

Data Fig. 4b), with 100\% concordance for SNP identity at positions 452, 484, and 501 for the control samples.

\section{Performance evaluation of the DETECTR ${ }^{\circledR}$ assay using clinical samples}

Next, we assembled a blinded dataset consisting of 93 COVID-19 positive clinical samples (previously analyzed by viral WGS) and the SNP controls run in parallel. These samples were extracted, amplified in triplicate RT-LAMP reactions (Extended Data Fig. 2), and processed further as triplicate CasDx1 reactions for each LAMP replicate (Extended Data Fig. 3). A total of nine replicates were thus generated for each sample to detect WT or MUT SNPs at positions 452,484 , and 501 . The DETECTR ${ }^{\circledR}$ data analysis pipeline was then applied to each sample to provide a final lineage categorization (Fig. 2a-c). For a biological RT-LAMP replicate to be designated as either WT or MUT, the same call needed to be made from all three technical CasDx1 replicates (Extended Data Fig. 5a). A final SNP mutation call was made based on $\geq 1$ of the same calls from the three biological replicates, with replicates that were designated as a No Call ignored (Extended Data Fig. 5a-c). After excluding two samples that were considered invalid because the fluorescence intensity from RT-LAMP amplification did not reach a pre-established threshold determined using receiver-operator characteristic (ROC) curve analysis (Extended Data Fig. 2 and Extended Data Fig. 6), we evaluated a total of 807 CasDx1 signals from the 91 remaining clinical samples, generating up to 9 replicates for each clinical sample (Extended Data Fig. 5b). Differentiation of WT and MUT signals according to the allele discrimination plots was more pronounced at positions 484 and 501 than position 452 (Extended Data Fig. 4), whereas the MA plots, 
medRxiv preprint doi: https://doi.org/10.1101/2021.11.29.21267041; this version posted February 3, 2022. The copyright holder for this preprint (which was not certified by peer review) is the author/funder, who has granted medRxiv a license to display the preprint in perpetuity. It is made available under a CC-BY-NC-ND 4.0 International license.

generated by transforming the data onto $\mathrm{M}$ (log ratio) and $\mathrm{A}$ (mean average) scales, showed clear separation of WT and MUT calls for all three positions (Fig. 3a and Extended Data Fig. 4). The variant calls made on each sample were consistent with the difference in median values of the log-transformed signals as determined using the data analysis pipeline (Extended Data Fig. 7). 
medRxiv preprint doi: https://doi.org/10.1101/2021.11.29.21267041; this version posted February 3, 2022. The copyright holder for this preprint (which was not certified by peer review) is the author/funder, who has granted medRxiv a license to display the preprint in perpetuity.

It is made available under a CC-BY-NC-ND 4.0 International license .

a

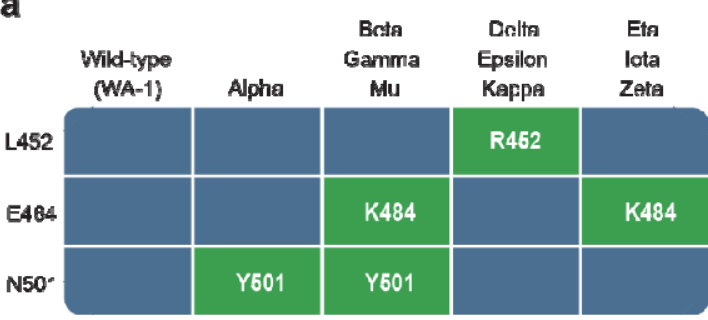

C

COVID-42

COVID-67

LAMP curves with threshold

COVID-2
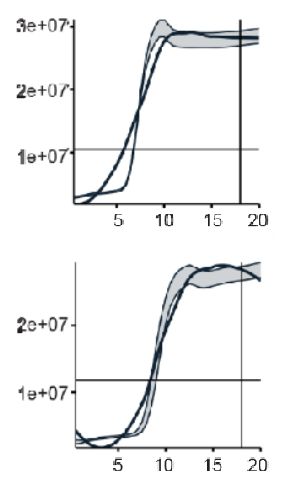

COVID-67

COVID-2
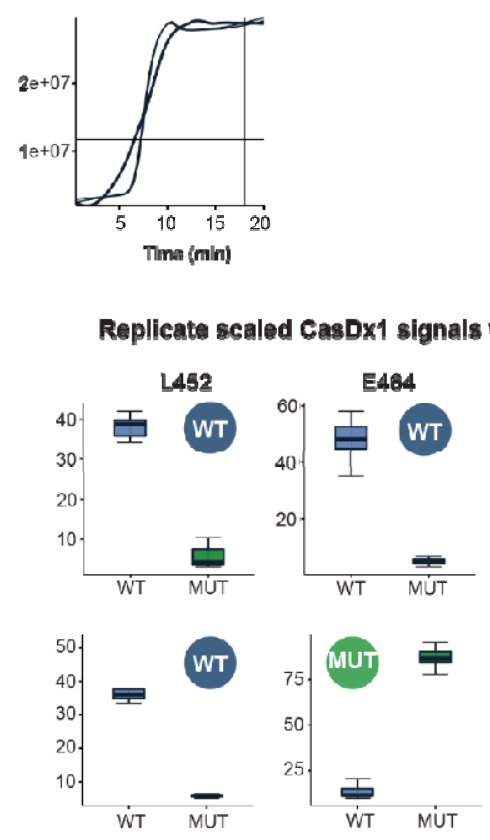

b

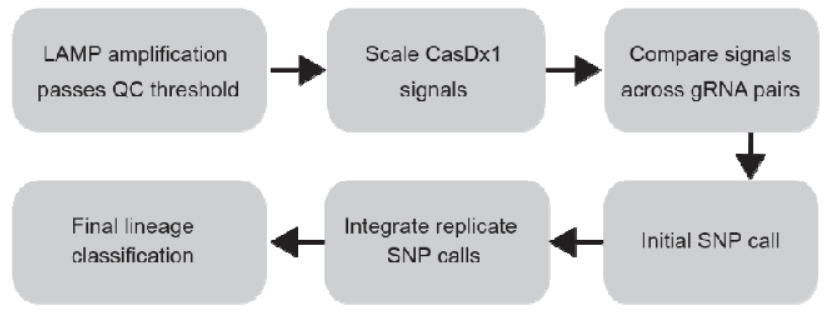

CasDx1 curves
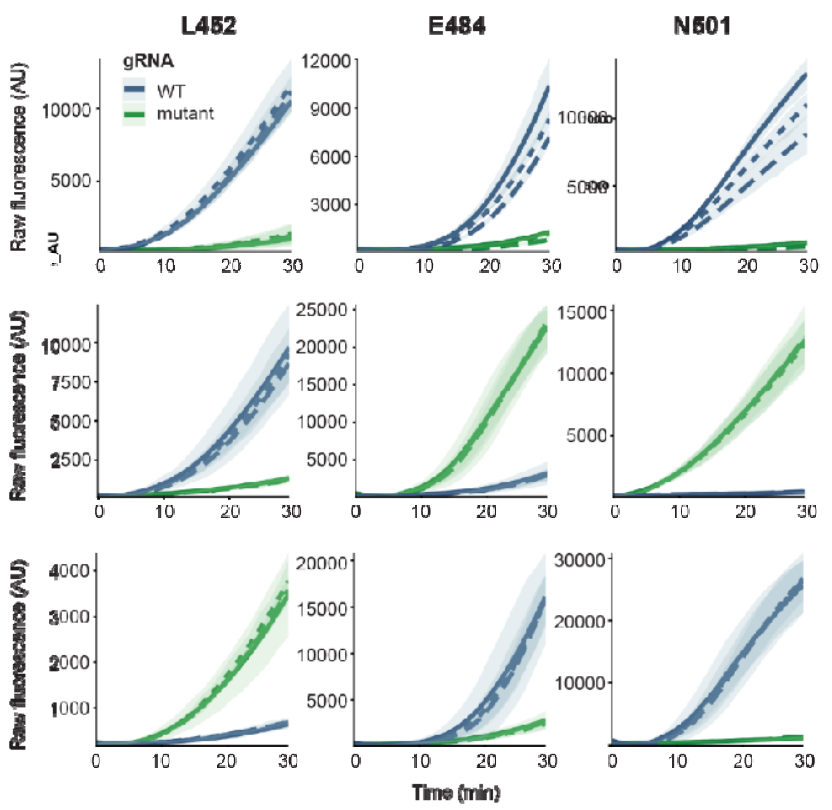

Replicate scaled CasDX1 signals with SNP calls

N501
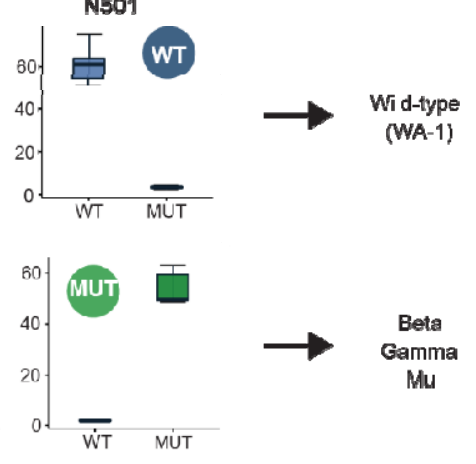

Variant classification
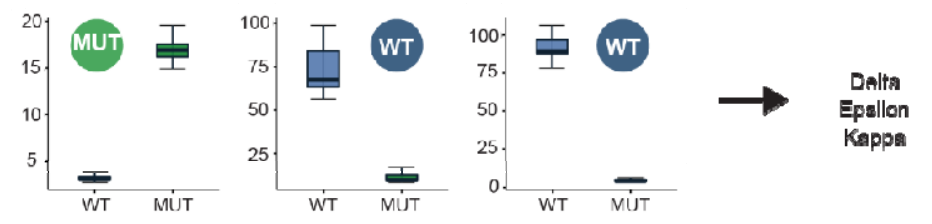


\section{Fig. 2 | DETECTR ${ }^{\circledR}$ data analysis pipeline for SARS-CoV-2 SNP mutation calling. a,} Interpretation table summarizing the SARS-CoV-2 mutations in this study associated with the corresponding lineage classification. b, Schematic of data analysis pipeline describing the RT-LAMP QC and subsequent CasDx1 signal scaling. The scaled signals were compared across SNPs and the calls were made for each RT-LAMP replicate. The combined replicate calls defined the mutation call, which informed the final lineage classification. c. Three representative clinical samples of different SARSCoV-2 lineages depict the workflow of the DETECTR ${ }^{\circledR}$ assay. Raw fluorescence curves of each sample run in RT-LAMP amplification and subsequent triplicate DETECTR ${ }^{\circledR}$ reactions targeting both WT and MUT SNPs for L452(R), E484(K), and N501(Y). Box plot visualization of the end point fluorescence in DETECTR ${ }^{\circledR}$ across each SNP for the three representative clinical samples. Calls were made for each SNP by evaluating the median values of the DETECTR ${ }^{\circledR}$ calls and overall calls through the LAMP replicates, and given a designation of WT, MUT, or NoCall. Final calls are made on the lineage determined by each SNP. Blue represents WT and green represents MUT, with RTLAMP replicates $(n=3)$, CasDx1 replicates ( $n=3$ per LAMP replicate) and shading around kinetic curves indicates $\pm 1.0 \mathrm{SD}$.

We then unblinded the viral WGS results to evaluate the accuracy of the DETECTR ${ }^{\circledR}$ assay for SNP calls and lineage classification. There were 14 discordant SNP calls out of 272 (94.9\% SNP concordance) distributed among 11 clinical samples out of 91 (Extended Data Fig. 8a-c). Among the 11 discordant samples, one sample (COVID-31) was designated a 'no call' at position 452 by viral WGS and thus lacked a comparator, 
two samples were designated a 'no call' due to flat WT and MUT curves (COVID-41 and COVID-73), four samples had similar WT and MUT curve amplitudes, suggesting a mixed population (COVID-03, COVID-56, COVID-61 and COVID-81) (Extended Data Fig. 8a), and four samples had SNP assignments discordant with those from viral WGS (COVID-12, COVID-13, COVID-20 and COVID-63) (Extended Data Fig. 8a).

Given that the comparison data had been collected over an extended time period, we surmised that sample stability issues arising from aliquoting and multiple freeze-thaw cycles may have accounted for the observed discrepancies. To further investigate this possibility, the 11 discordant clinical samples were re-extracted from the original respiratory swab matrix and re-analyzed by running both viral WGS and the DETECTR ${ }^{\circledR}$ assay in parallel. Re-testing of the samples resulted in nearly complete agreement between the two methods, except for two SNPs that were identified as E484Q in two samples by WGS but were incorrectly called E484 (WT) by the DETECTR ${ }^{\circledR}$ assay (Fig. 3b-c and Extended Data Fig. 8d). Thus, based on discrepancy testing, the positive predictive agreement (PPA) between the DETECTR ${ }^{\circledR}$ assay and viral WGS at all three WT and MUT SNP positions was $100 \%$ (272 of 272, $\mathrm{p}<2.2 \mathrm{e}-16$ by Fisher's Exact Test) (Fig. 3d). The corresponding negative predictive agreement (NPA) was $91.4 \%$ as the E484Q mutation for two SNPs was incorrectly classified as WT. Nevertheless, the final viral lineage classification for the 91 samples after discrepancy testing showed 100\% agreement with viral WGS (Fig. 3d and Supplementary Table 1). 
a

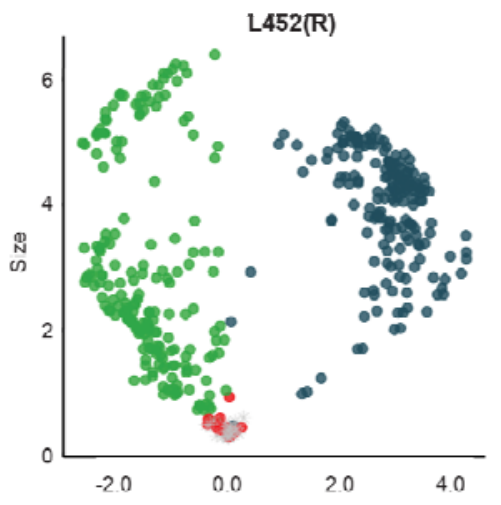

b

$$
\text { was }
$$

s:
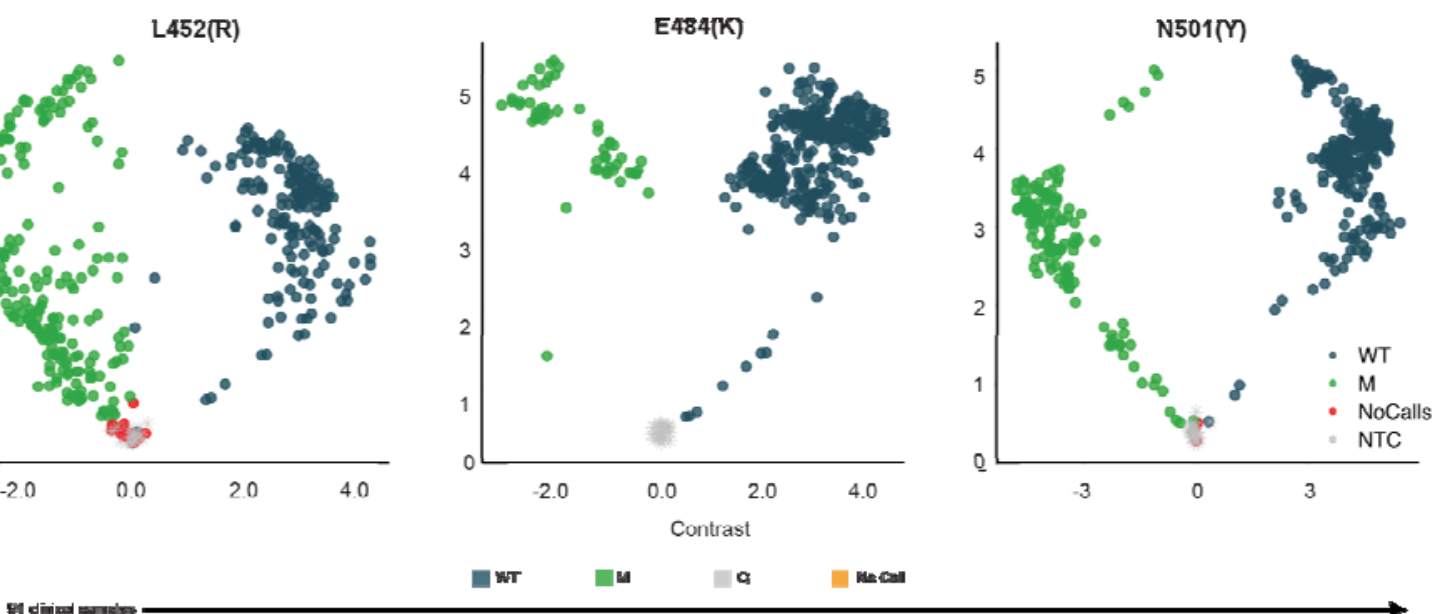
DETECTR:

$\mathbf{E}$ garoonew

$$
\text { 베 }
$$

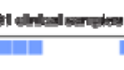

andan

d

\begin{tabular}{|c|c|c|c|c|}
\hline \multirow{5}{*}{$\begin{array}{c}\text { coviD-19 } \\
\text { Variant } \\
\text { DETECTR } \circledast 8\end{array}$} & & \multicolumn{3}{|c|}{ SARS-CoV-2 WGS } \\
\hline & N501(Y) & WT & MUT & Tota \\
\hline & WT & 67 & 0 & 57 \\
\hline & MUT & 0 & 34 & 34 \\
\hline & Total & 67 & 34 & 91 \\
\hline \multicolumn{2}{|c|}{ PPA N501 (WT) } & \multicolumn{3}{|c|}{$500 x$} \\
\hline \multicolumn{2}{|c|}{ NPA N501 (WT) } & \multicolumn{3}{|c|}{$100 \%$} \\
\hline \multicolumn{2}{|c|}{ PPA Y501 (MUT) } & \multicolumn{3}{|c|}{1008} \\
\hline \multicolumn{2}{|c|}{ NPA Y501 (MUT) } & \multicolumn{3}{|c|}{$160 \%$} \\
\hline
\end{tabular}

\begin{tabular}{|c|c|c|c|c|}
\cline { 3 - 5 } \multicolumn{2}{c|}{} & \multicolumn{3}{c|}{ SARS-CoV-2 WGS } \\
\hline \multirow{3}{*}{$\begin{array}{c}\text { COVID-19 } \\
\text { Variant } \\
\text { DETECTR }\end{array}$} & L452(R) & WT & MUT & Total \\
\cline { 2 - 5 } & WT & 22 & 0 & 42 \\
\cline { 2 - 5 } & MUT & 0 & 48 & 48 \\
\hline & Total & 2 & 48 & 00 \\
\hline \multicolumn{2}{|c|}{ PPA L452 (WT) } & \multicolumn{3}{|c|}{$100 \%$} \\
\hline \multicolumn{2}{|c|}{ NPA L452 (WT) } & \multicolumn{3}{|c|}{$100 \%$} \\
\hline \multicolumn{2}{|c|}{ PPA R452 (MUT) } & \multicolumn{3}{|c}{} \\
\hline \multicolumn{2}{|c|}{ NPA R452 (MUT) } & \multicolumn{3}{|c}{} \\
\hline
\end{tabular}

\begin{tabular}{|c|c|c|c|c|c|}
\hline \multirow{5}{*}{$\begin{array}{c}\text { COVID-19 } \\
\text { Variant } \\
\text { DETECTR }\end{array}$} & & \multicolumn{4}{|c|}{ SARS-CoV-2 WGS } \\
\hline & $\mathrm{E} 484(\mathrm{~K})$ & WT & MUT & $\mathbf{Q}$ & Total \\
\hline & WT & 68 & 0 & $2^{*}$ & 70 \\
\hline & MUT & 0 & 21 & 0 & 21 \\
\hline & Total & 68 & 21 & 2 & 91 \\
\hline \multicolumn{2}{|c|}{ PPA E484 (WT) } & \multicolumn{4}{|c|}{$100 \%$} \\
\hline \multicolumn{2}{|c|}{ NPA E484 (WT) } & \multicolumn{4}{|c|}{$91.3 \%$} \\
\hline \multicolumn{2}{|c|}{ PPA K484 (MUT) } & \multicolumn{4}{|c|}{$100 \%$} \\
\hline \multicolumn{2}{|c|}{ NPA K484 (MUT) } & \multicolumn{4}{|c|}{$100 \%$} \\
\hline
\end{tabular}

\begin{tabular}{|c|c|c|c|}
\cline { 2 - 4 } \multicolumn{1}{c|}{} & \multicolumn{3}{c|}{ Overall SNP concordance } \\
\hline SNP & WT & MUT & p-value \\
\hline 452 & $100 \%$ & $100 \%$ & $2.20-18$ \\
\hline 484 & $100 \%$ & $100 \%$ & $<2.20-16$ \\
\hline 501 & $100 \%$ & $100 \%$ & $2.20-16$ \\
\hline
\end{tabular}

\section{Fig. 3 | Comparison of the DETECTR ${ }^{\circledR}$ assay to SARS-CoV-2 Whole-Genome}

Sequencing. a, MA plots, transformed onto M (log ratio) and A (mean average) scales, show CasDx1 SNP detection replicates $(n=807)$ for each SARS-CoV-2 
mutation across 91 clinical samples. WT is denoted by blue dots, MUT is denoted by green dots, NoCall is denoted by orange dots and NTC is denoted by grey dots. $\mathbf{b}$, Alignment of final mutation calls comparing the DETECTR ${ }^{\circledR}$ and SARS-CoV-2 WGS assay results across 91 clinical samples after discordant samples (indicated by red asterisk) were resolved. c, Final lineage classification on each clinical sample by the DETECTR $^{\circledR}$ assay compared to the SARS-CoV-2 lineage determined by viral WGS. d, Final Positive Predictive Agreement (PPA), Negative Predictive Agreement (NPA) and concordance values for each WT and MUT SNP from the evaluation of the DETECTR ${ }^{\circledR}$ assay against the SARS-CoV-2 WGS comparator assay after discordant samples were resolved.

In November 2021, a new SARS-CoV-2 variant was identified and almost immediately designated a variant of concern, called Omicron ${ }^{39}$. The Omicron variant carries an exceptionally high number of mutations $(>30)$ within the S-gene and has been shown to have enhanced transmissibility and immune evasion ${ }^{40,41}$. The record number of COVID19 cases globally from Omicron and loss of activity by certain therapeutic antibodies underscores the need for rapid and targeted identification of SARS-CoV-2 variants. Although the TaqPath PCR assay with S-gene Target Failure (SGTF) has functioned as a screen that can be reflexed to sequencing to identify the Omicron variant ${ }^{42}$, the SGTF assay alone cannot differentiate between Omicron BA. 1 and Alpha ${ }^{43,44}$ and cannot identify emerging variants that lack the SGTF, such as the Omicron BA.2 sublineage ${ }^{43}$. We therefore quickly reconfigured our COVID-19 variant DETECTR ${ }^{\circledR}$ assay for the identification of Omicron by targeting the E484A mutation, which alone differentiates 
Omicron from all other current VBM/VOI/VOC. Given that E484-related mutations are present in multiple circulating variants and have a strong effect on reducing antibody neutralization ${ }^{10,45,46}$, we further updated our panel of CasDx1 gRNAs to detect all relevant mutations (E, K, Q and A) at amino acid position 484 (Fig. 4a-b).

Given the highly mutated Omicron S-gene, we suspected that our original LAMP primer set would not have sufficient sensitivity to amplify the targeted spike RGD region and thus we incorporated degenerate nucleotides within the LAMP primers to enable amplification of the Omicron S-gene (Fig. 4a, Extended Data Fig. 9, and Supplementary Table 3). Within weeks of the first Omicron case identified in the U.S. ${ }^{47}$, we procured and tested an additional set of 48 clinical samples. These samples were blinded and processed with the updated DETECTR ${ }^{\circledR}$ assay workflow, which included sample extraction, followed by amplification with the degenerate LAMP primers and detection with each of the 484-specific CasDx1 gRNAs. Once processed, a result with mutations K484, Q484 or A484 was called Beta/Eta/Gamma/lota/Mu/Zeta, Kappa, or Omicron, respectively (Fig. 4c-e and Extended Data Fig. 10a). If the result was associated with E484 (WT), we ran the assay using WT and MUT gRNAs at positions 452 and 501 to call the final SARS-CoV-2 lineage (Fig. 4c-e and Extended Data Fig. 10a). Using this workflow, we detected 36 out of 48 total clinical samples: $18 / 48$ resulted as E484 (WT) and were subsequently tested with 452 and 501 gRNAs (3/18 called WT, 6/18 called Alpha and 9/18 called Delta), 4/48 resulted as K484 (called Beta/Eta/Gamma/lota/Mu/Zeta), 2/48 resulted as Q484 (called Kappa), and 12/48 resulted as A484 (called Omicron) (Fig. 4e and Extended Data Fig. 10b-c). The 
remaining 12/48 clinical samples neither amplified nor showed any DETECTR ${ }^{\circledR}$ signal and were thus called "Not Detected" (Supplementary Table 2).

Unblinding of samples COVID-92 through COVID-127 revealed five discordant samples: COVID-103, COVID-108, COVID-109, COVID-112 and COVID-122 (Extended Data Fig. 10d). All five discordant samples were re-extracted from the original patient sample and re-processed with WGS and COVID Variant DETECTR ${ }^{\circledR}$. After repeat testing, three samples (COVID-103, COVID-108, COVID-109) showed 100\% concordance between WGS and DETECTR ${ }^{\circledR}$, with both methods resulting in "No Call” at position 452. Notably, these samples were also part of the original set of 91 samples (COVID-20, COVID-63, COVID-73) that were previously concordant at position 452, suggesting a decrease in sample integrity likely resulting from multiple freeze/thaw cycles incurred during several re-extractions. Sample COVID-112 was called an Omicron by DETECTR ${ }^{\circledR}$ based on its A484 SNP call, which was confirmed by WGS. Finally, sample COVID-122 could not be amplified by RT-LAMP, also suggesting a loss in sample integrity. Following this discrepancy analysis, we demonstrated an overall SNP concordance of $94.7 \%$, and $100 \%$ NPA for this set of 48 samples (Fig. 4 e). 
medRxiv preprint doi: https://doi.org/10.1101/2021.11.29.21267041; this version posted February 3, 2022. The copyright holder for this preprint (which was not certified by peer review) is the author/funder, who has granted medRxiv a license to display the preprint in perpetuity.

It is made available under a CC-BY-NC-ND 4.0 International license .

a

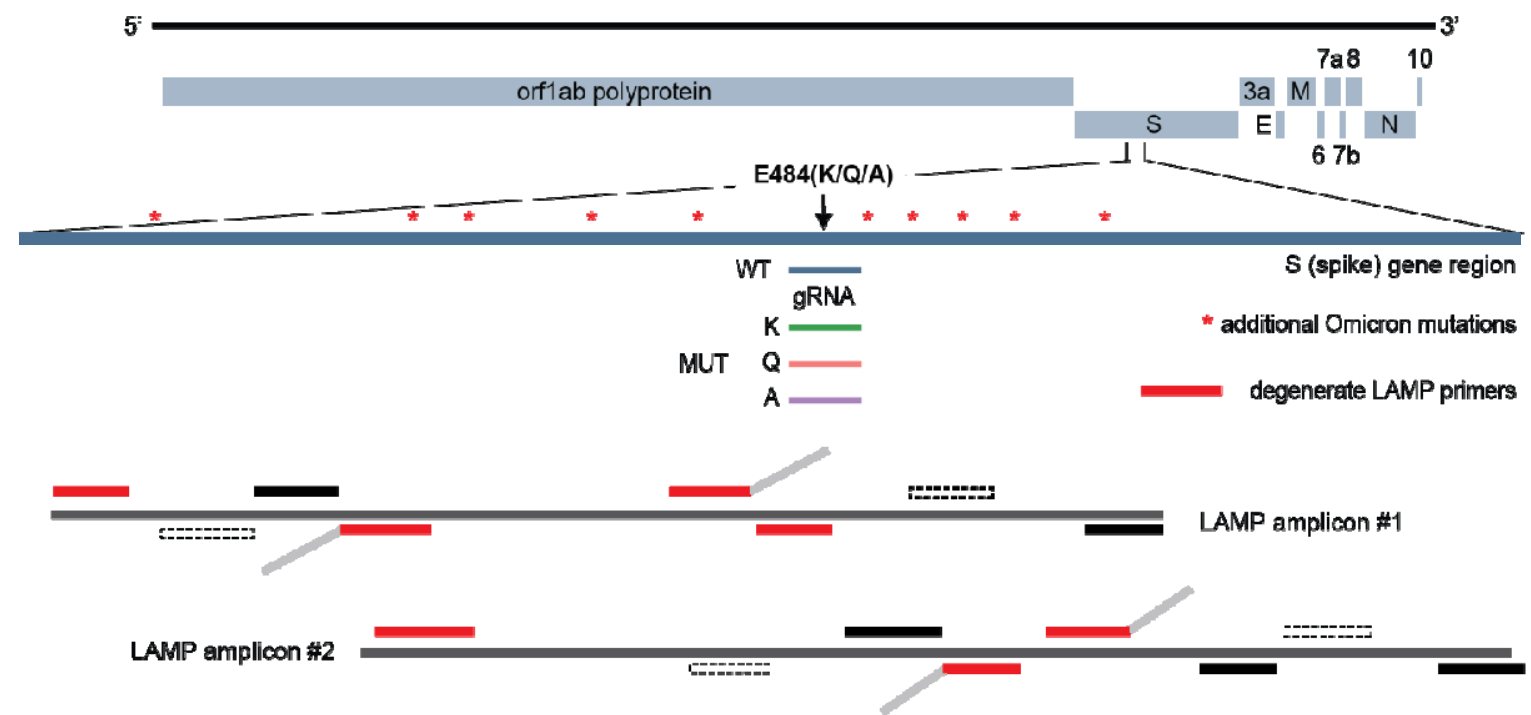

b

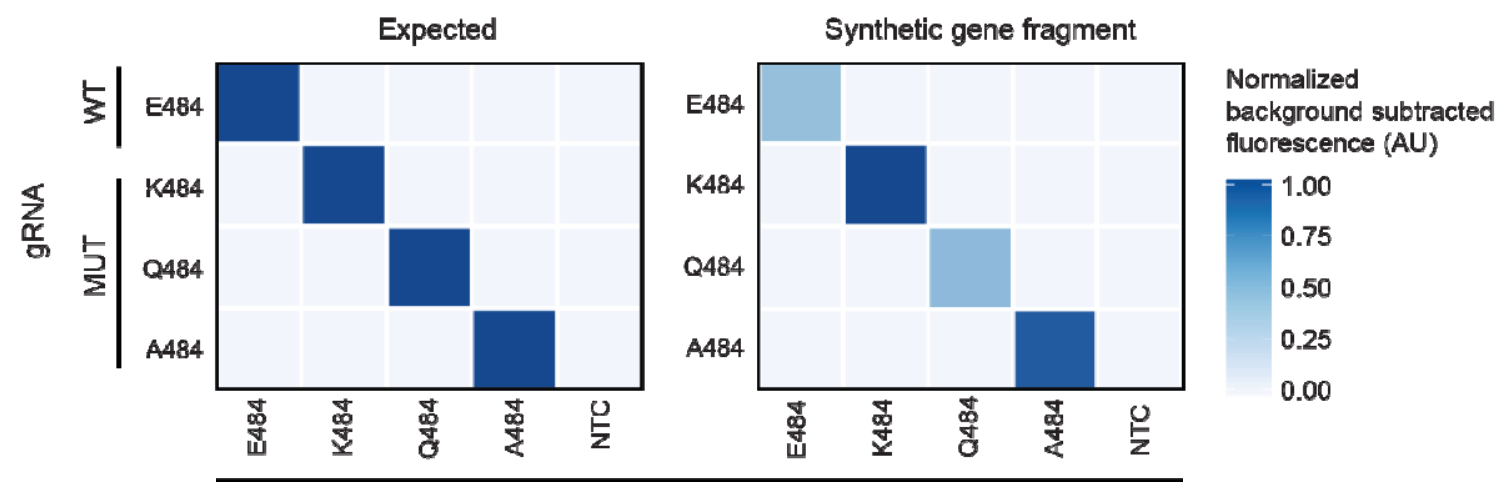

Target mutation

C

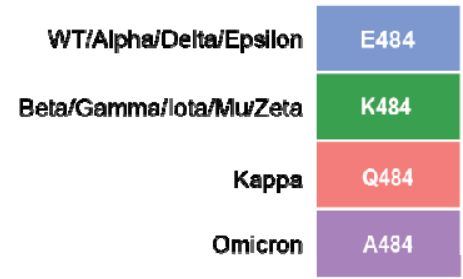

e

\begin{tabular}{|c|c|c|}
\hline SNP & Concordance & p-value \\
\hline E484 & $\mathbf{9 4 . 7} \%$ & $<1.88 \mathrm{E}-15$ \\
\hline K484 & $\mathbf{1 0 0} \%$ & $<1.88 \mathrm{E}-15$ \\
\hline Q484 & $\mathbf{1 0 0} \%$ & $<1.88 \mathrm{E}-15$ \\
\hline A484 & $100 \%$ & $<1.88 \mathrm{E}-15$ \\
\hline
\end{tabular}

d

WTIApha/Delta/Epsi on Beta/Gamma/loba/Muzzeta

Kapps

Omieron Indeberminant 
Fig. 4 | Specific detection of 484 mutations enables rapid Omicron identification.

a, Schematic of Omicron mutations within the S-gene LAMP amplicon and relative position of 484-specific gRNAs and degenerate LAMP primers. b, Heat map comparison of end-point fluorescence $(t=30 \mathrm{~min})$ showing specific detection of 484specific mutations (E, K, Q, A) on PCR-amplified synthetic gene fragments $(n=3)$. c, SARS-CoV-2 lineage classification table based on 484 mutations. d, Alignment of final 484 mutation calls comparing the DETECTR ${ }^{\circledR}$ and SARS-CoV-2 WGS assay results across 36 clinical samples. e, Overall SNP concordance values for the 484 SNP from the evaluation of the DETECTR ${ }^{\circledR}$ assay against the SARS-CoV-2 WGS comparator assay.

\section{Discussion}

In this study, we developed a CRISPR-based DETECTR ${ }^{\circledR}$ assay for the detection of SARS-CoV-2 variants. We evaluated three CRISPR-Cas 12 enzymes, two commercially available (LbCas12a from NEB and AsCas12a from IDT) and one proprietary (CasDx1 from Mammoth Biosciences). Based on a head-to-head comparison of these enzymes, we observed clear differences in performance, with CasDx1 demonstrating the highest fidelity as the only enzyme able to reliably detect all targeted SNPs. A data analysis pipeline, developed to differentiate between WT and MUT signals with the DETECTR ${ }^{\circledR}$ assay, yielded an overall SNP concordance of $97.9 \%$ (373/381 total SNP calls) and 99.3\% (138/139) agreement with lineage classification compared to viral WGS. These findings show robust agreement between the DETECTR ${ }^{\circledR}$ assay and viral WGS for 
identification of SNP mutations and variant categorization. Thus, the DETECTR ${ }^{\circledR}$ assay provides a faster and simpler alternative to sequencing-based methods for COVID-19 variant diagnostics and surveillance.

Our results show that the choice of Cas enzyme is important to maximize the accuracy of CRISPR-based diagnostic assays and may need to be tailored to the site that is being targeted. As currently configured with the L452R, E484K/Q/A and N501Y SNP targets, the COVID-19 variant DETECTR ${ }^{\circledR}$ assay is currently capable of distinguishing the Alpha, Delta, Kappa and Omicron variants, but cannot resolve the remaining VBMs or VOIs. However, given the rapid emergence and shifts in the distribution of variants over time $\mathrm{e}^{13}$ it is likely that tracking of key mutations, many of which are suspected to arise from convergent evolution ${ }^{48}$, rather than tracking of variants, will be more important for surveillance as the pandemic continues. Here we also developed a data analysis pipeline for CRISPR-based SNP calling that can readily incorporate additional targets and offers a blueprint for automated interpretation of fluorescent signal patterns.

Although CRISPR-based diagnostic assays have been previously demonstrated for the detection of SARS-CoV-2 variants, these studies have limitations regarding coverage of circulating lineages, the extent of clinical sample evaluation, and/or assay complexity. For example, the miSHERLOCK variant assay uses LbCas12a (NEB) with RPA preamplification to detect N501Y, E484K and Y144Del covering eight lineages (WA-1, Alpha, Beta, Gamma, Eta, lota, Mu and Zeta) and was tested only on contrived samples (RNA spiked into human saliva) ${ }^{21}$. The SHINEv2 assay uses LwaCas13a with RPA pre- 
amplification to detect 69/70Del, K417N/T, L452R and 156/157Del + R158G covering eight lineages (WA-1, Alpha, Beta, Gamma, Delta, Epsilon, Kappa and Mu) and was tested with only the 69/70Del gRNAs on 20 Alpha-positive NP clinical samples ${ }^{49}$. Finally, the mCARMEN variant identification panel (VIP) uses 26 crRNA pairs with either the LwaCas13a or LbaCas13a and PCR pre-amplification to identify all current circulating lineages including Omicron; however, the VIP requires the Fluidigm Biomark HD system or similar, more complex instrumentation for streamlined execution ${ }^{50}$. In comparison, the DETECTR ${ }^{\circledR}$ assay presented here uses CasDx1 with LAMP preamplification to detect N501Y, E484K/Q/A and L452R covering all current circulating lineages including Omicron and tested on 139 clinical samples representing eight lineages (WA-1, Alpha, Gamma, Delta, Epsilon, lota, Mu, Omicron). Furthermore, we demonstrate here that specific Omicron identification can be accomplished using only the E484 WT and A484 MUT guides.

Some limitations of our study are as follows. First, as previously mentioned, the DETECTR ${ }^{\circledR}$ assay currently detects only the L452R, E484K/Q/A and N501Y mutations, which may not provide enough resolution to identify future lineages. Second, we observed variable performance of the assay in SNP discrimination, with more potential overlap in the calls between WT and MUT for the 452 position than for the other two sites. These two limitations could potentially be addressed by the incorporation of additional gRNAs to the assay to provide specific and redundant coverage and to improve identification of specific lineages. Third, due to a multiplexed and degenerate S-gene LAMP primer design, the limit of detection of the DETECTR ${ }^{\circledR}$ assay is higher 
than our previously published SARS-CoV-2 DETECTR ${ }^{\circledR}$ assay $^{20}$, and thus only positive clinical samples with a $\mathrm{Ct}<30$ (near the limit of detection for viral whole-genome sequencing) were tested in our study. Incorporation of an additional $\mathrm{N}$-gene target to the assay may be necessary if simultaneous detection and SNP/variant identification is desired. Finally, the current study focuses on the development and validation of a variant DETECTR ${ }^{\circledR}$ assay using conventional laboratory equipment. Future work will involve implementation onto automated, portable systems for use in point of care settings.

In the near term, we suggest the use of the DETECTR ${ }^{\circledR}$ assay as an initial screen for circulating variants and/or a distinct pattern from a rare or novel variant by interrogating the key 452,484 , and 501 positions that could be reflexed to viral WGS. As the sequencing capacity for most clinical and public health laboratories is limited, the DETECTR ${ }^{\circledR}$ assay would thus enable rapid identification of variants circulating in the community to support outbreak investigation and public health containment efforts. Identification of specific mutations associated with neutralizing antibody evasion ${ }^{12,51}$ could inform patient care with regards to the use of monoclonal antibodies that remain effective in treating the infection ${ }^{15}$. As the virus continues to mutate and evolve, the DETECTR $^{\circledR}$ assay can be readily reconfigured by validating new gRNAs and preamplification LAMP primers and gRNAs that target emerging mutations with clinical and epidemiological significance. Over the longer term, a validated CRISPR assay that combines SARS-CoV-2 detection with variant identification offers a faster and simpler alternative to sequencing and would be useful as a tool for simultaneous COVID-19 
medRxiv preprint doi: https://doi.org/10.1101/2021.11.29.21267041; this version posted February 3, 2022. The copyright holder for this preprint (which was not certified by peer review) is the author/funder, who has granted medRxiv a license to display the preprint in perpetuity. It is made available under a CC-BY-NC-ND 4.0 International license.

diagnosis in individual patients and surveillance for infection control and public health purposes. 


\section{Materials and Methods}

\section{Synthetic Gene Fragments}

Wild-type (WT) and mutant (MUT) synthetic gene fragments (Twist) were PCR amplified using NEB 2x Phusion Master Mix following the manufacturer's protocol. The amplified product was cleaned using AMPure XP beads following manufacturers protocol at a $0.7 x$ concentration. The product was eluted in nuclease-free water and normalized to 10 nM. All nucleic acids used in this study are summarized in Supplementary Table 3.

\section{Clinical sample acquisition and extraction}

De-identified residual SARS-CoV-2 RT-PCR positive nasopharyngeal and/or oropharyngeal (NP/OP) swab samples in universal transport media (UTM) or viral transport media (VTM) were obtained from the UCSF Clinical Microbiology Laboratory. All samples were stored in a biorepository according to protocols approved by the UCSF Institutional Review Board (protocol number 10-01116, 11-05519) until processed.

All NP/OP swab samples obtained from the UCSF Clinical Microbiology Laboratory were pretreated with DNA/RNA Shield (Zymo Research, \# R1100-250) at a 1:1 ratio. The Mag-Bind Viral DNA/RNA 96 kit (Omega Bio-Tek, \# M6246-03) on the KingFisher Flex (Thermo Fisher Scientific, \# 5400630) was used for viral RNA extraction using an input volume of $200 \mu \mathrm{l}$ of diluted NP/OP swab sample and an elution volume of $100 \mu \mathrm{l}$. 
medRxiv preprint doi: https://doi.org/10.1101/2021.11.29.21267041; this version posted February 3, 2022. The copyright holder for this preprint (which was not certified by peer review) is the author/funder, who has granted medRxiv a license to display the preprint in perpetuity.

It is made available under a CC-BY-NC-ND 4.0 International license .

The Taqpath ${ }^{\text {TM }}$ COVID-19 RT-PCR kit (Thermo Fisher Scientific) was used to determine the $\mathrm{N}$ gene cycle threshold values.

\section{Heat-inactivated culture acquisition and extraction}

Heat-inactivated cultures of SARS-CoV-2 Variants Being Monitored (VBM), Variants of

Concern (VOC) or Variants of Interest (VOI) were provided by the California Department of Public Health (CDPH).

RNA from heat-inactivated SARS-CoV-2 VBM/VOC/VOI isolates were extracted using the EZ1 Virus Mini Kit v2.0 (Qiagen, \# 955134) on the EZ1 Advanced XL (Qiagen, \# 9001875) according to the manufacturer's instructions. For each culture, six replicate LAMP reactions were pooled into a single sample. DETECTR ${ }^{\circledR}$ was performed on a 1:10 dilution of the $10,000 \mathrm{cp} / \mathrm{rxn}$ LAMP amplification products.

\section{COVID-19 variant DETECTR ${ }^{\circledR}$ assay}

Two LAMP primer sets, each containing 6 primers, were designed to target the L452R, E484K and N501Y mutations in the SARS-CoV-2 Spike (S) protein (Supplemental Table). Sets of LAMP primers were designed from a 350 bp target sequence spanning the 3 mutations using Primer Explorer V5 (https://primerexplorer.jp/e/). Candidate primers were manually evaluated for inclusion using the OligoCalc online oligonucleotide properties calculator ${ }^{52}$ while ensuring that there was no overlap with either primers from the other set or guide RNA target regions that included the L452R, E484K, and N501Y mutations. 
Multiplexed RT-LAMP was performed using a final reaction volume of $50 \mu \mathrm{l}$, which consisted of $8 \mu \mathrm{l}$ RNA template, $5 \mu \mathrm{l}$ of L452R primer set (Eurofins Genomics), $5 \mu \mathrm{l}$ of E484K/N501Y primer set, $17 \mu \mathrm{l}$ of nuclease-free water, $1 \mu$ of SYTO-9 dye (ThermoFisher Scientific), and $14 \mu \mathrm{l}$ of LAMP mastermix. Each of the primer sets consisted of $1.6 \mu \mathrm{M}$ each of inner primers FIP and BIP, $0.2 \mu \mathrm{M}$ each of outer primers F3 and B3, and $0.8 \mu \mathrm{M}$ each of loop primers LF and LB). The LAMP mastermix contained 6 $\mathrm{mM}$ of $\mathrm{MgSO}_{4}$, isothermal amplification buffer at $1 \mathrm{X}$ final concentration, $1.5 \mathrm{mM}$ of dNTP mix (NEB), 8 units of Bst 2.0 WarmStart DNA Polymerase (NEB), and $0.5 \mathrm{ul}$ of WarmStart RTx Reverse Transcriptase (NEB). Plates were incubated at $65^{\circ} \mathrm{C}$ for 40 minutes in a real-time Quantstudio ${ }^{\text {TM }} 5$ PCR instrument. Fluorescent signals were collected every 60 seconds

Degenerate multiplexed RT-LAMP was performed using a final reaction volume of $65 \mu \mathrm{l}$, which consisted of $9.6 \mu \mathrm{l} \mathrm{RNA}$ template, $10 \mu \mathrm{l}$ of L452R degenerate primer set (Eurofins Genomics), $10 \mu \mathrm{l}$ of E484K/N501Y degenerate primer set, $14.1 \mu \mathrm{l}$ of nuclease-free water, $1.3 \mu$ of SYTO-9 dye (ThermoFisher Scientific), and $20 \mu$ of LAMP mastermix.The primer set and mastermix assembly, the incubation and data collection were described above.

40nM CasDx1 (Mammoth Biosciences), LbCas12a (EnGen ${ }^{\circledR}$ Lba Cas12a, NEB) or AsCas12a (Alt- ${ }^{\circledR}$ A.s. Cas12a, IDT) protein targeting the WT or MUT SNP at L452(R), E484(K) or N501(Y) was incubated with 40nM gRNA in 1X buffer (MBuffer3 for CasDx1, 
NEBuffer r2.1 for LbCas12a and AsCas12a) for 30 min at $37^{\circ} \mathrm{C}$. Dx1 gRNAs were used with both CasDx1 and LbCas12a, whereas AsCas12a gRNAs were used with AsCas12a (Supplementary Table 3). 100nM ssDNA reporter (/5Alex594N/TTATTATT/3IAbRQSp/, IDT) was added to the RNA-protein complex. $18 \mu \mathrm{L}$ of this DETECTR ${ }^{\circledR}$ master mix was combined with $2 \mu \mathrm{L}$ target amplicon. The DETECTR ${ }^{\circledR}$ assays were monitored for $30 \mathrm{~min}$ at $37^{\circ} \mathrm{C}$ in a plate reader (Tecan).

\section{Digital PCR}

Samples were evaluated at 3 dilutions $(1: 100 ; 1: 1,000$; and 1:10,000) using the ApexBio Covid-19 Multiplex Digital PCR Detection Kit (Stilla Technologies) according to the manufacturer's protocol. The controls (positive and negative provided by UCSF, the Kit Controls, and an internal control) were run with the samples in duplicate. The dilutions were used to determine the most accurate concentration which was determined from the $\mathrm{N}$ gene concentration.

\section{Sequencing methods}

Complementary DNA (cDNA) synthesis from RNA via reverse transcription and tiling multiplexed amplicon PCR were performed using SARS-CoV-2 primers version 3 according to the Artic protocol ${ }^{53,54}$. Libraries were constructed by ligating adapters to the amplicon products using NEBNext Ultra II DNA Library Prep Kit for Illumina (New England Biolabs, \# E7645L), barcoding using NEBNext Multiplex Oligos for Illumina (New England Biolabs, \# E6440L), and purification with AMPure XP (Beckman-Coulter, 
\# 63880). Final pooled libraries were sequenced on either Illumina Miseq or NextSeq 550 as $2 \times 150$ single-end reads (300 cycles).

SARS-CoV-2 viral genome assembly and variant analyses were performed using an inhouse bioinformatics pipeline. Briefly, sequencing reads generated by Illumina sequencers (MiSeq or NextSeq 550) were demultiplexed and converted to FASTQ files using bcl2fastq (v2.20.0.422). Raw FASTQ files were first screened for SARS-CoV-2 sequences using BLASTn (BLAST+ package 2.9.0) alignment against the Wuhan-Hu-1 SARS-CoV-2 viral reference genome (NC_045512). Reads containing adapters, the ARTIC primer sequences, and low-quality reads were filtered using BBDuk (version 38.87) and then mapped to the NC_045512 reference genome using BBMap (version 38.87). Variants were called with CallVariants and iVar (version 1.3.1) and a depth cutoff of 5 was used to generate the final assembly. Pangolin software (version 3.1.17) $)^{55,56}$ was used to identify the lineage. Using a custom in-house script, consensus FASTA files generated by the genome assembly pipeline were scanned to confirm L452R, E484K, and N501Y mutations.

\section{Discordant sample retesting}

The discordant samples $(n=16)$ were re-extracted as described above for the NP/OP swab samples and evaluated by viral WGS as described above. The extracted nucleic acids were then thawed (incurring an additional freeze/thaw as needed) and amplified using the LAMP protocol described above and evaluated using the DETECTR ${ }^{\circledR}$ assay as described above. 


\section{DETECTR $^{\circledR}$ data analysis pipeline}

\section{Quality Control Metric for the LAMP Reaction}

Prior to processing DETECTR ${ }^{\circledR}$ data from the clinical samples, we collected data indicating the success or failure of the samples to amplify in the LAMP reaction. The absolute truth was based on visual inspection of LAMP curves This absolute truth was used to develop thresholds for the LAMP reactions. The positive and negative controls from the LAMP reactions were used to derive the thresholds to qualify the samples. Two sets of thresholds were used: time threshold and fluorescence rate threshold. The positive LAMP controls were assumed to represent an ideal sample and displayed a classic sigmoidal rise of fluorescence over time and the NTC represented the background fluorescence. It was hypothesized that a sample will ideally have positive control like fluorescence kinetics. However, due to the presence of high background in some samples, a mean value between controls for each plate was chosen as threshold. After this, the fluorescence values at a time threshold of 18 minutes were collected. The time point is of importance here to rule out those samples that would amplify closer to the endpoint, signifying the LAMP intermediates to be the majority contributors of the rise in the signal and not the actual sample itself. A score was assigned for each sample which was calculated as a ratio of rate of fluorescence rate threshold to the rate of fluorescence value at 18 minutes for each sample. The hypothesis is that if this ratio of rate of fluorescence between controls and samples is less than 1, then samples have failed to reach the minimum fluorescence required to be called out as amplified and if the ratio is greater than or equal to 1 , then samples have amplified sufficiently. To 
identify the exact score value for a qualitative QC metric, an ROC analysis was done on scores and the absolute truth (Extended Data Fig. 6).

\section{Data Analysis for CRISPR-based SNP calling}

Each well had a guide specific to the mutant or the wild-type SNP. The comparison is important to assign a genotypic call to the sample. The DETECTR ${ }^{\circledR}$ reactions across the plate are not comparable to each other. For this purpose, the endpoint fluorescence intensities are normalized in each well to its own minimum intensity. This term is called fluorescence yield. The fluorescence yield was compared across wells in a plate under the assumption that each well would have similar minimum fluorescence starting point. Irrespective of the highest levels of the fluorescence intensities observed across samples, the yield for a given target must ideally remain the same assuming that similar concentrations of samples/target are being compared. This aided in normalizing the signal and comparing replicates across the wells in the same plate.

$$
F_{y}=\max (F) / \min (F)
$$

The wild-type and mutant target guides on NTC must ideally not show any change in intensity over time. The fluorescence yield for NTC must remain constant across replicates, plates and close to 1 .

$$
F_{y}(N T C)=1
$$

On the contrary, if a sample has a fluorescence yield of 1 , then it qualifies for a No Call.

General rules for variant calling

1. NTC was assigned NTC 
2. If the Contrast of the sample for a SNP was between minimum and maximum contrast for the plate, then the sample is assigned a NoCall.

3. If the Size of the sample is lower than the Size of the NTC on the plate, then the sample is assigned a NoCall.

$$
\begin{gathered}
C_{\min }(N T C \text {-snp })<=C(\text { sample-snp })<=C_{\max }(N T C-\text { snp }) \rightarrow \text { NoCall } \\
S_{\min }(N T C \text {-snp })<=S(\text { sample-snp })<=S_{\max }(N T C-\text { snp }) \rightarrow \text { NoCall } \\
\log 2(F y(W T))>\log 2(F y(M)) \rightarrow \text { Wild Type } \\
\log 2(F y(W T))<\log 2(F y(M)) \rightarrow \text { Mutant }
\end{gathered}
$$

In cases, where more than one mutant at a particular position was being analyzed for, then the signals of each mutant was compared with the wild-type signal. If there existed a mutant, then among $(\mathrm{n})$ comparisons for $\mathrm{n}$ mutants and one wild type, one of the comparisons would yield mutant call.

$$
\begin{aligned}
& \log 2(F y(W T))>\log 2(F y(M 1)) \rightarrow \text { Wild Type } \\
& \log 2(F y(W T))>\log 2(F y(M 2)) \rightarrow \text { Wild Type } \\
& \log 2(F y(W T))>\log 2(F y(M 3)) \rightarrow \text { Wild Type } \\
& \log 2(F y(W T))<\log 2(F y(M 4)) \rightarrow \text { Mutant }(4)
\end{aligned}
$$


If there was a tie in the above logic between mutant and wild-type, then a tie breaker comparison would yield a final result.

$$
\begin{aligned}
& \log 2(F y(W T))>\log 2(F y(M 1)) \rightarrow \text { Wild Type } \\
& \log 2(F y(W T))<\log 2(F y(M 2)) \rightarrow \text { Mutant(2) } \\
& \log 2(F y(W T))>\log 2(F y(M 3)) \rightarrow \text { Wild Type } \\
& \log 2(F y(W T))<\log 2(F y(M 4)) \rightarrow \text { Mutant (4) } \\
& \log 2(F y(M 2))<\log 2(F y(M 4)) \rightarrow \text { Mutant(4) }
\end{aligned}
$$

\section{$\underline{\text { SNP Calls }}$}

We used the following procedure to evaluate the concordance between sequencing and DETECTR $^{\circledR}$ technologies for genotypic classification of the clinical cohort dataset. First, we considered all samples and SNPs for which both sequencing and DETECTR ${ }^{\circledR}$ data was present in the distributed files by matching the SNP IDs and sample names. This included cleaning and curing the dataset which had failed LAMP reactions and identifying WT and MUT based on the spacer fluorescent. This yielded a preliminary data set containing 279 calls across three SNPs against 93 samples. After eliminating samples that had failed to amplify in the LAMP reaction but were assigned a genotype, the resulting final analysis data consisted of 272 calls (WT, MUT and NoCall) spread across three SNPs and 91 samples. For each of the three SNPs in the analysis data set, we identified and recorded both sequencing and DETECTR ${ }^{\circledR}$ genotypes (including NoCalls and LAMP Fails) for each of the 93 patients. The 91 patients include the individuals for whom actual sequencing data was available 


\section{Statistical analysis}

\section{SNP Calls}

For each SNP in the analysis, we computed a variety of statistics evaluating the concordance between genotype calls on the two different technologies. The concordant and discordant genotypes were visualized through contingency tables. For each SNP, there are three possible genotypes (WT, MUT and No Call). The concordance rates were calculated without the samples that failed the LAMP reaction (Fig. 3B and Supplementary Table 1). The $2 \times 2$ cross tables classify all three SNPs across all the samples between sequencing and DETECTR ${ }^{\circledR}$ technologies (Fig. 3B and

Supplementary Table 1). The data transformation and statistical analysis was done in $\mathrm{R}^{57}$.

\section{Acknowledgments}

We thank the UCSF Center for Advanced Technology core facility (Delsy Martinez and Tyler Miyasaki) for their efforts in high-throughput sequencing of viral cDNA libraries using the Illumina NovaSeq 6000 instrument, and Mary Kate Morris from the California Department of Public Health for providing the heat-inactivated viral cultures. We also thank Lucas Harrington and Teresa Peterson for their critical review of this manuscript.

This work has been funded by the Innovative Genomics Institute (IGI) at UC Berkeley and UC San Francisco (C.Y.C.), the Sandler Program for Breakthrough Biomedical Research (C.Y.C.), US Centers for Disease Control and Prevention contract 75D30121C10991 (C.Y.C.), and Mammoth Biosciences. 


\section{Author contributions}

C.L.F., J.S.C., and C.Y.C. conceived and designed the study. C.Y.C, and V.S. coordinated the SARS-CoV-2 whole-genome sequencing efforts and RT-LAMP primer design and testing. C.L.F., J.P.B., J.C., and J.S.C. designed guide RNAs for CRISPRCas12 testing. B.M., J.P.B., R.N.D., E.S., and C.G.H. tested guide RNAs and ran DETECTR $^{\circledR}$ experiments. V.S., N.B., B.W., A.S.-G., K.R., J.S., S.M., and C.Y.C. collected samples. C.L.F., V.S., B.M., V.N., J.P.B., J.C., J.S.C., and C.Y.C. analyzed data. C.L.F., V.S., V.N., B.M., J.P.B., J.C., J.S.C., and C.Y.C. wrote the manuscript. All authors read the manuscript and agree to its contents.

\section{Author Declarations}

I confirm all relevant ethical guidelines have been followed, and any necessary IRB and/or ethics committee approvals have been obtained.

The details of the IRB/oversight body that provided approval or exemption for the research described are given below: Remnant nasopharyngeal and/or oropharyngeal (NP/OP) samples and plasma samples from laboratory confirmed SARS-CoV-2 positive patients were retrieved from the UCSF Clinical Laboratories and stored in a biorepository until processed. Remnant sample biobanking was performed with a waiver of consent and according to no-subject contact study protocols approved by the UCSF Institutional Review Board (protocol numbers 10-01116 and 11-05519). 
I confirm that all necessary patient/participant consent has been obtained and the appropriate institutional forms have been archived, and that any patient/participant/sample identifiers included were not known to anyone (e.g., hospital staff, patients or participants themselves) outside the research group so cannot be used to identify individuals.

I understand that all clinical trials and any other prospective interventional studies must be registered with an ICMJE-approved registry, such as ClinicalTrials.gov. I confirm that any such study reported in the manuscript has been registered and the trial registration ID is provided (note: if posting a prospective study registered retrospectively, please provide a statement in the trial ID field explaining why the study was not registered in advance).

I have followed all appropriate research reporting guidelines and uploaded the relevant EQUATOR Network research reporting checklist(s) and other pertinent material as supplementary files, if applicable.

\section{Competing interests}

C.Y.C. is the director of the UCSF-Abbott Viral Diagnostics and Discovery Center and receives research support from Abbott Laboratories, Inc. C.L.F., B.M., V.N., J.P.B., R.N.D., E.S., C.G.H., J.C., and J.S.C. are employees of Mammoth Biosciences. C.Y.C. is a member of the scientific advisory board for Mammoth Biosciences. The other authors declare no competing interests. 


\section{Data and Materials Availability}

All data needed to evaluate the conclusions in the paper are present in the paper and/or the Supplementary Materials. The CasDx1 protein can be provided by Mammoth Biosciences to the extent feasible, pending scientific review and a completed material transfer agreement. Requests for the CasDx1 protein should be submitted to Janice Chen at janice@mammothbiosci.com. 


\section{Supplementary Figures}
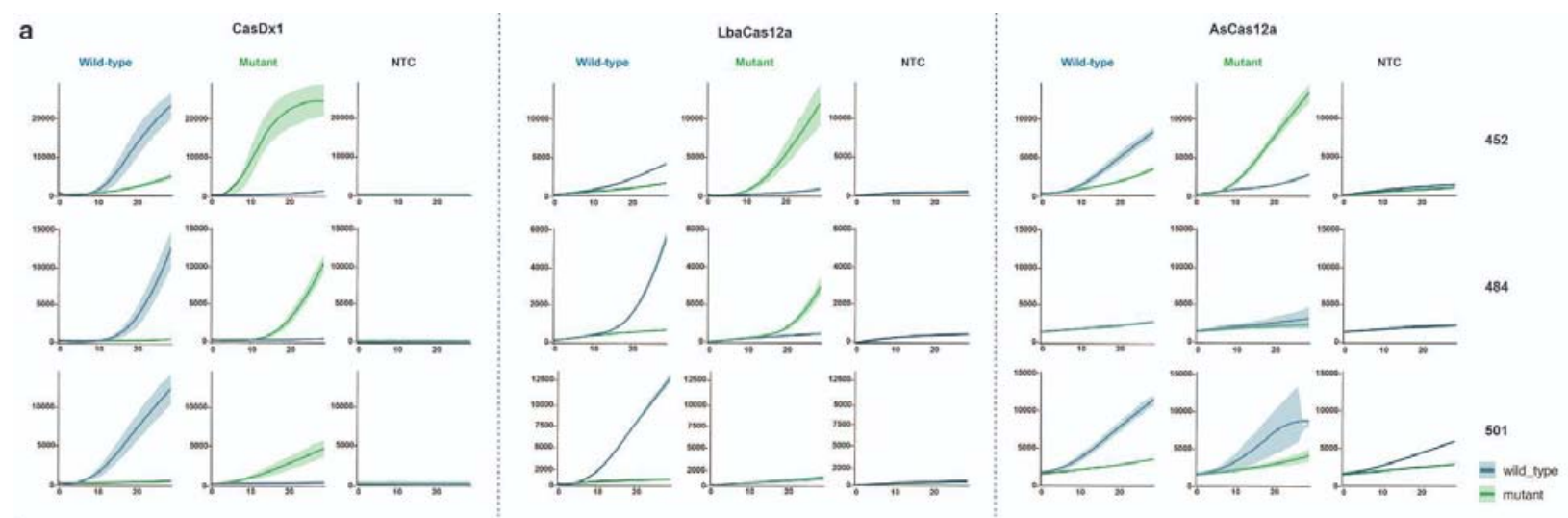

b
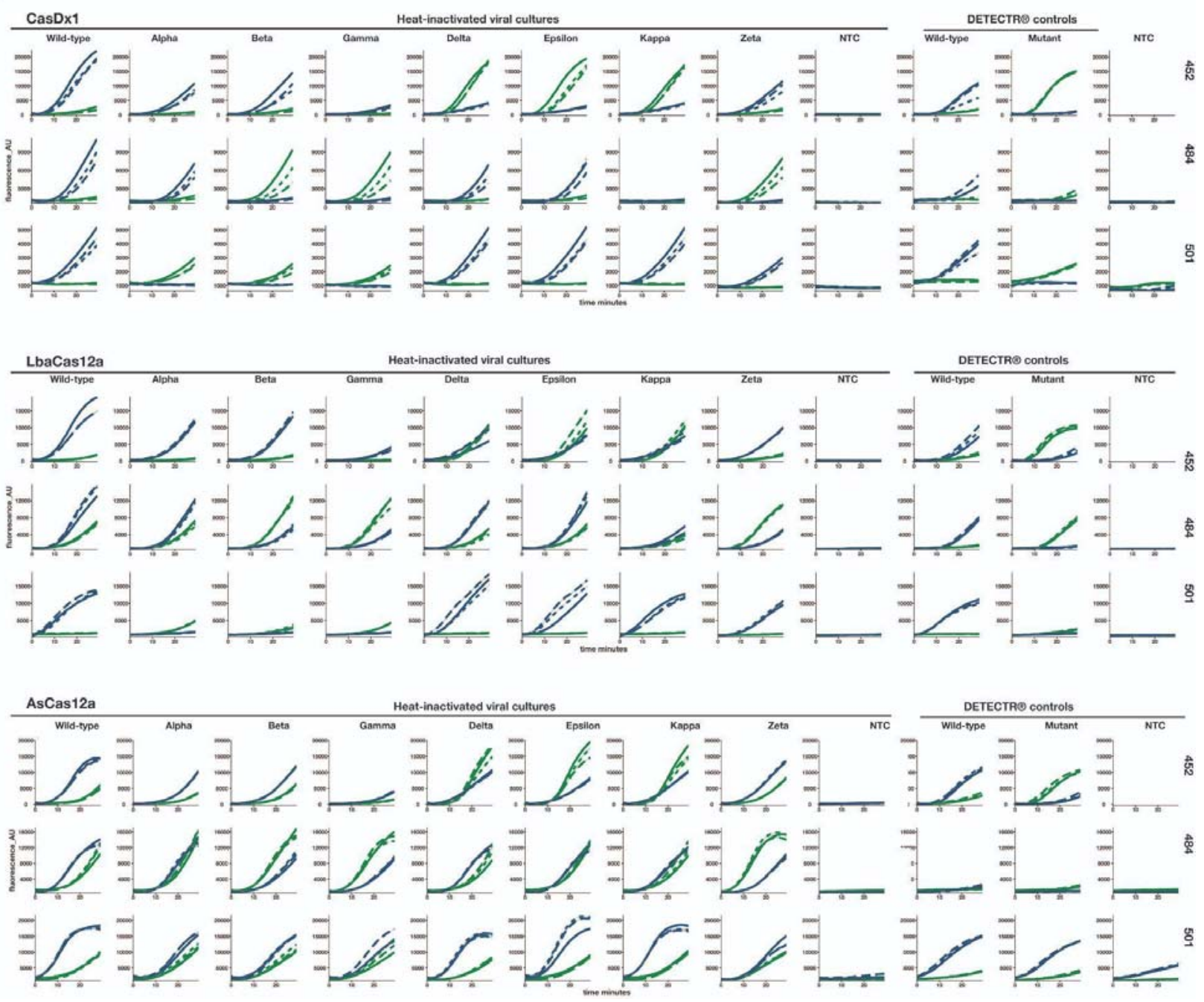
medRxiv preprint doi: https://doi.org/10.1101/2021.11.29.21267041; this version posted February 3, 2022. The copyright holder for this preprint (which was not certified by peer review) is the author/funder, who has granted medRxiv a license to display the preprint in perpetuity.

It is made available under a CC-BY-NC-ND 4.0 International license .

\section{Extended Data Fig. 1 | DETECTR ${ }^{\circledR}$ curves from gene fragments and heat-}

inactivated viral cultures. a, Raw fluorescence curves from three Cas 12 enzymes

(CasDx1, LbCas12a, AsCas12a) complexed with WT and MUT SNP gRNAs run on

PCR-amplified gene fragments representing WT and MUT SNP targets. $\mathbf{b}$, Raw

fluorescence curves from three Cas12 enzymes (CasDx1, LbCas12a, AsCas12a) on

eight heat-inactivated viral culture samples from various SARS-CoV-2 lineages, a no target control (RT-LAMP) and CasDx1 detection controls (WT, MUT and NTC). CasDx1 replicates $(n=3), \pm 1.0 S D$.
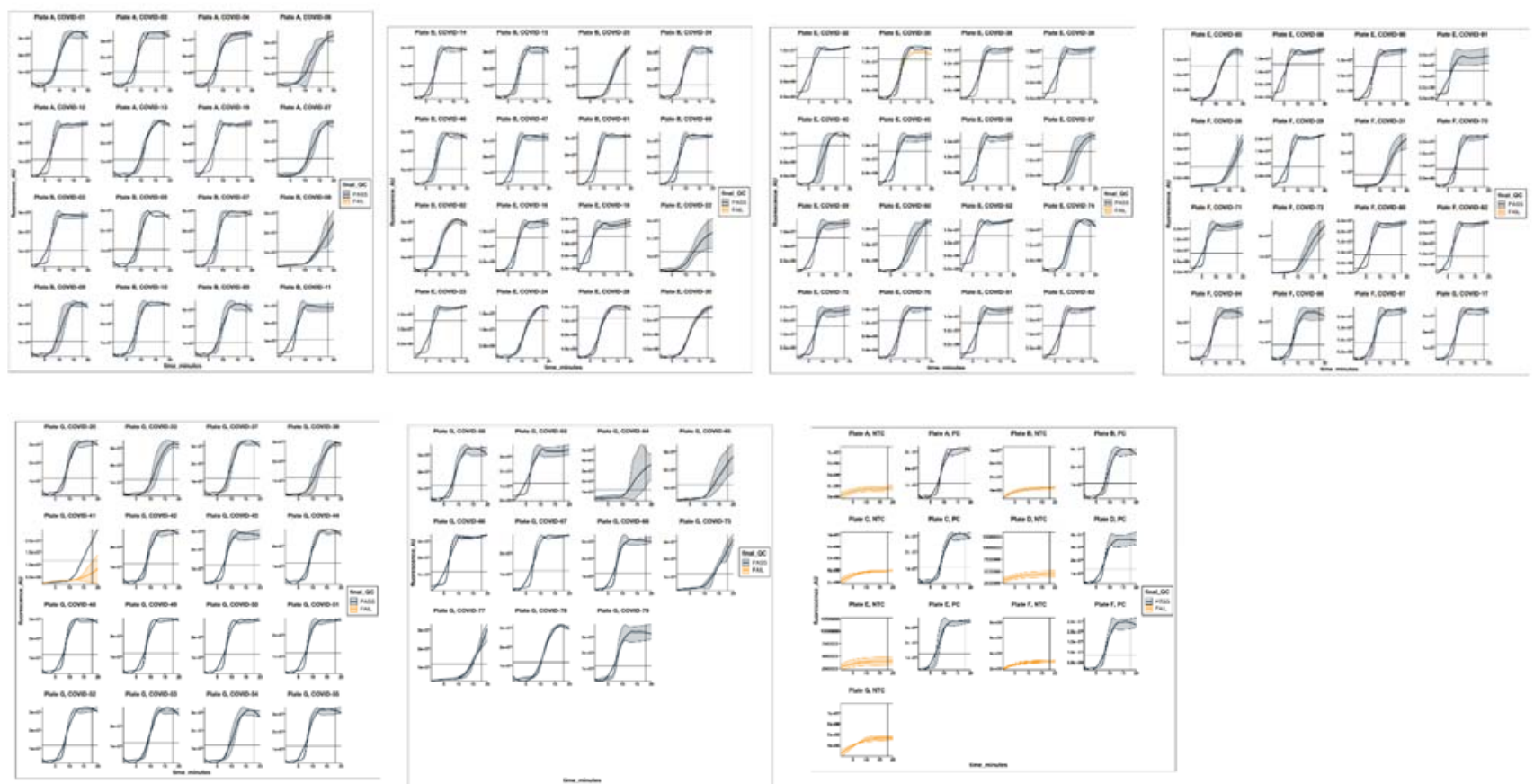

Extended Data Fig. 2 | Raw fluorescence RT-LAMP curves for each clinical 
sample. The raw fluorescence RT-LAMP amplification curves for each of the clinical samples analyzed ( $n=3$ replicates). Each line is representative of the median \pm 1.0 SD of the three RT-LAMP replicates for each sample. RT-LAMP replicates that passed QC are represented in navy blue and failed LAMP replicates are shown in orange. Only valid RT-LAMP replicates were used in subsequent data analysis.

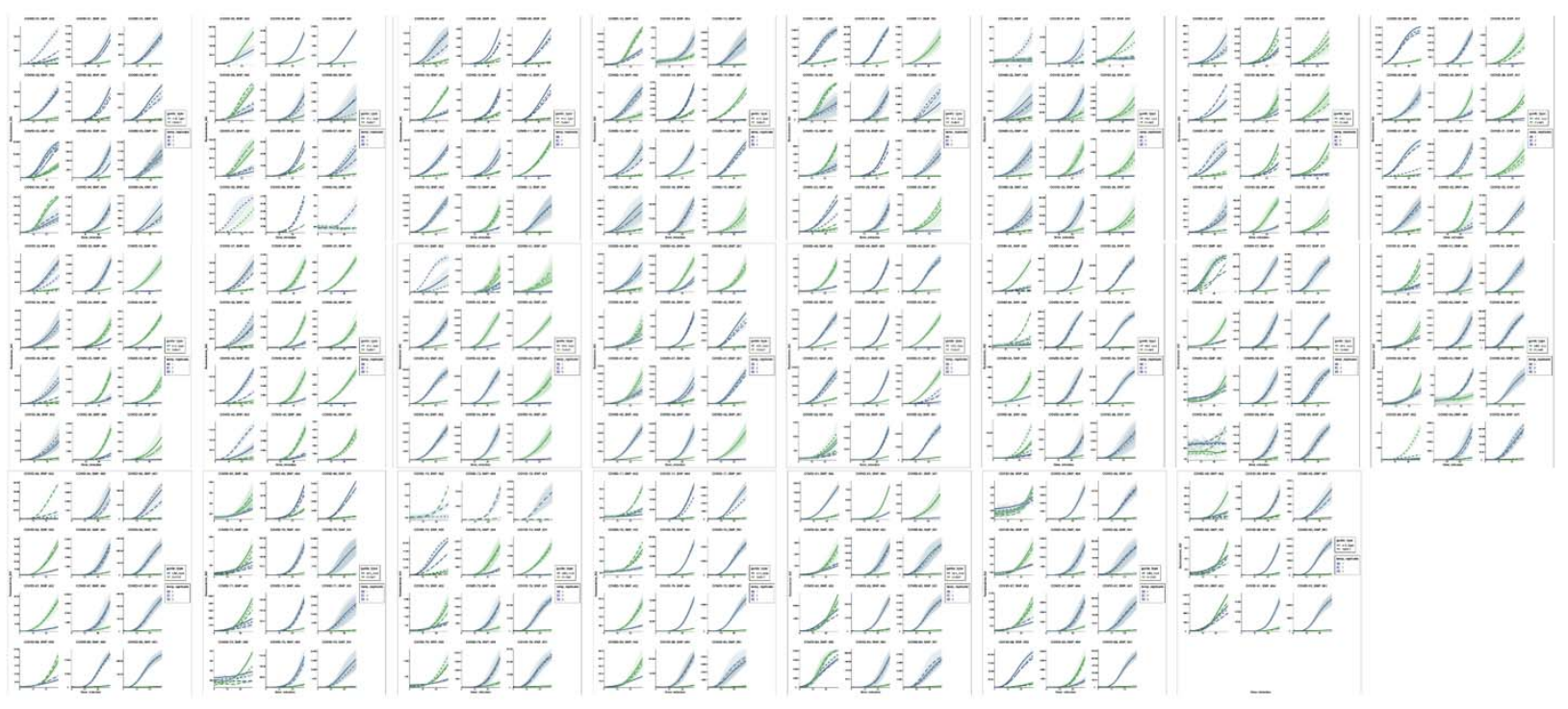

\section{Extended Data Fig. 3 | Raw fluorescence CasDx1 curves for each clinical sample}

amplified by RT-LAMP. Each clinical sample was amplified with RT-LAMP in triplicate, and the resulting amplicons were detected by CasDx1 in triplicate. The raw fluorescence curves show WT detection in blue and MUT detection in green. Each line is representative of the median \pm 1.0 SD of the CasDx1 replicates $(n=3)$ for each WT and MUT guide for each of the RT-LAMP replicates $(n=3)$, represented by different patterned lines. 

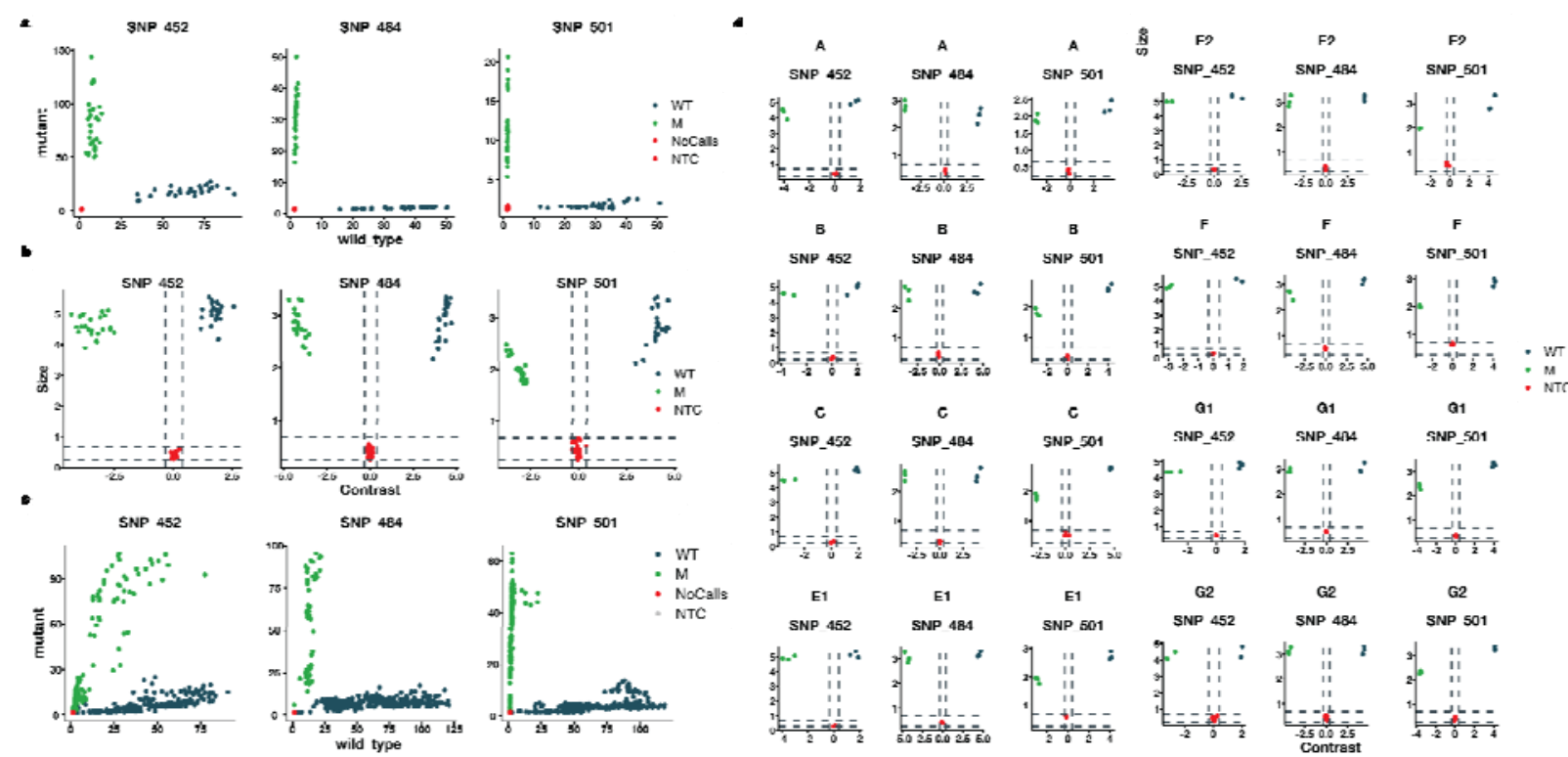

\section{Extended Data Fig. 4 | Evaluation of the DETECTR ${ }^{\circledR}$ data analysis pipeline and}

making final calls. a, Allele discrimination plot visualizing the scaled signals from the COVID Variant DETECTR ${ }^{\circledR}$ assay on gene fragments. The allele discrimination plots represent scatter plots of scaled WT and MUT fluorescence values plotted against each other. b, Contrast-Size plots of the COVID Variant DETECTR ${ }^{\circledR}$ assay data on gene fragments to decrease ambiguity of the scaled signals, a ratio of the WT and MUT transformed values are plotted against the average of the WT and MUT transformed values on the MA plot. c, Allele discrimination plot visualizing the scaled signals from the COVID Variant DETECTR ${ }^{\circledR}$ assay on clinical sample. $d$, MA plots of the COVID Variant DETECTR ${ }^{\circledR}$ assay on the gene fragments $(n=30$ WT; $n=30$ MUT for each SNP) and no template controls ( $n=33$ WT; $n=33$ MUT for each SNP) used to test the data analysis. 
a

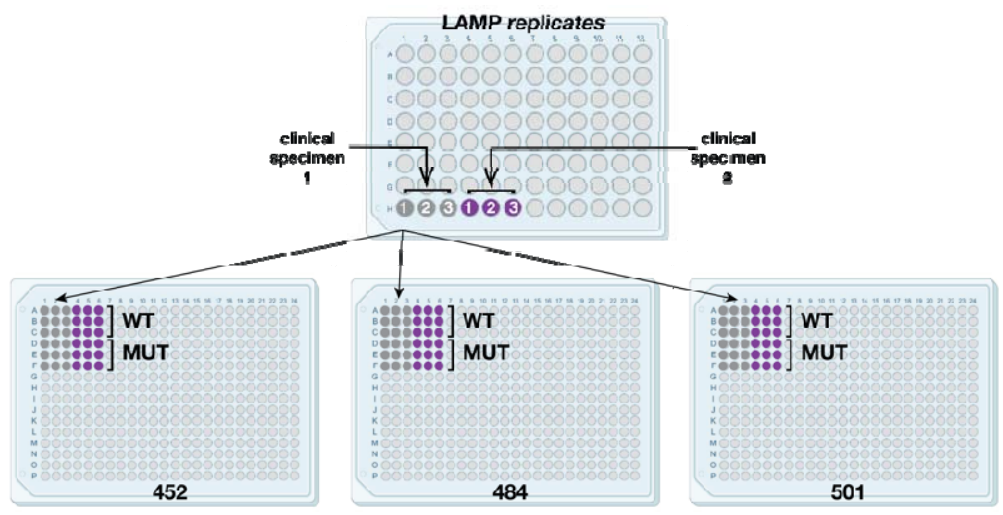

b

Clinical apecimen - 1

Clinical opecimen - 2

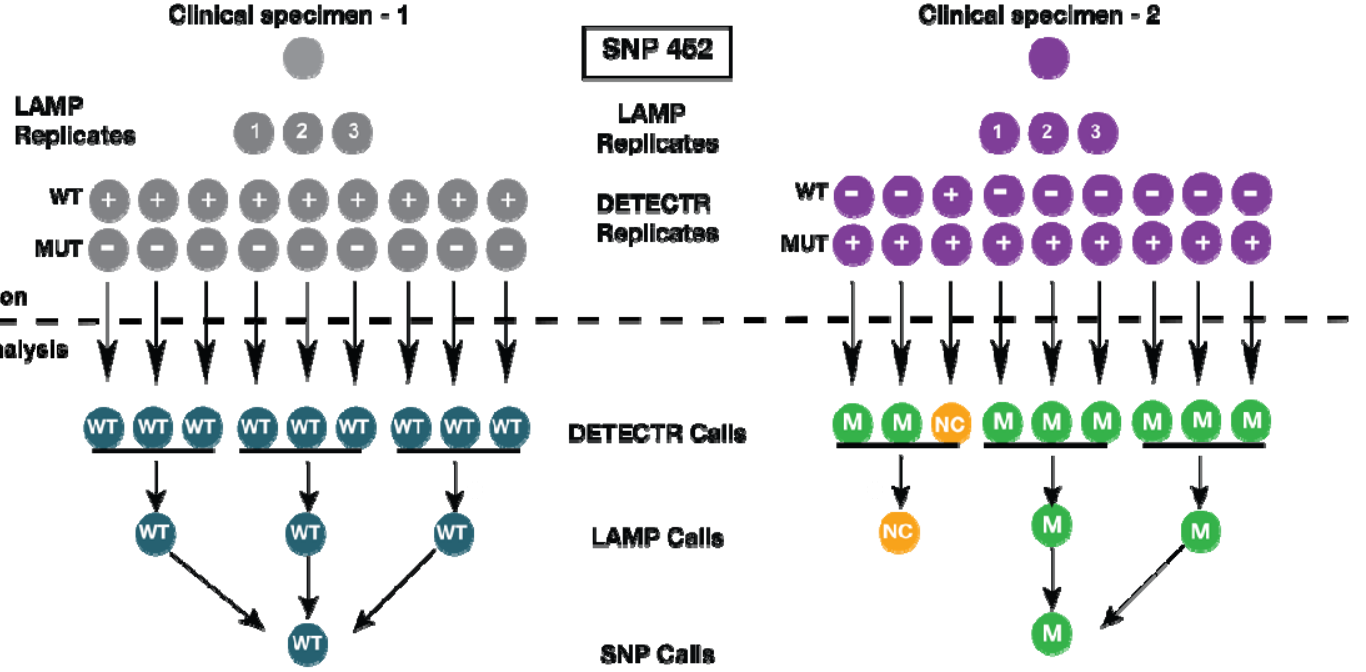

c

Data collection Algorithm analye

SNP Cails

Lase medleater

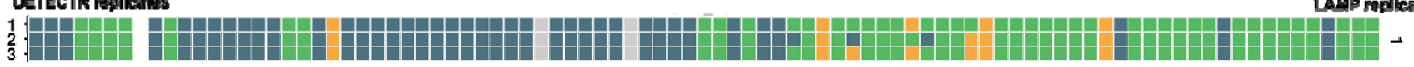

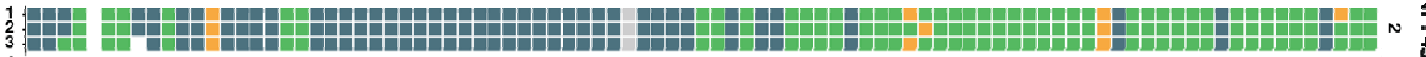

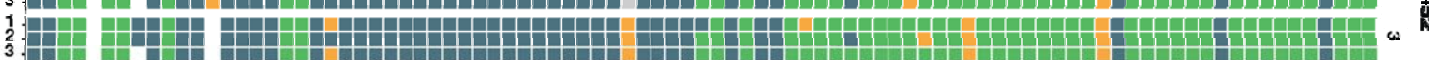

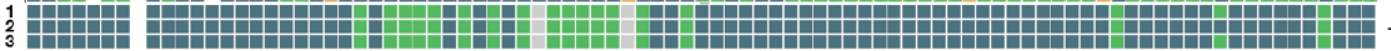

1
2

1
2

2
1

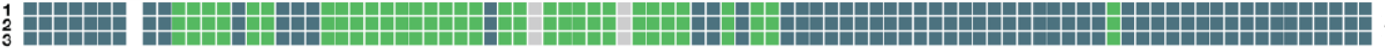

1
2
3

1 -

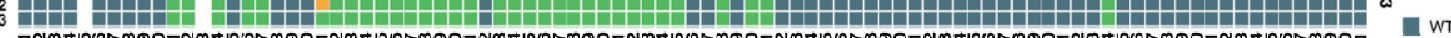

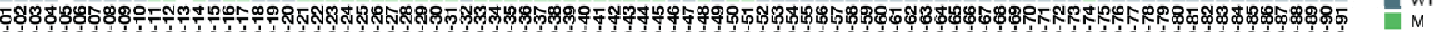

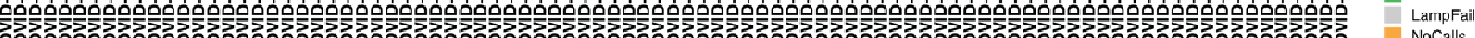

¿000

Extended Data Fig. 5 | Highly specific detection by CasDx1 for each SNP on RT-

LAMP replicates from clinical samples. a, The DETECTR ${ }^{\circledR}$ assay workflow from

LAMP amplification to SNP identification. $\mathbf{b}$, Schematic showing the relationship 
between clinical samples, LAMP replicates and CasDx1 replicates that culminate in a final SNP call. c, Heat map showing CasDx1 signal $(n=3)$ per every LAMP replicate $(n$ = 3) for each SNP on every clinical sample reflecting samples prior to discordance testing.

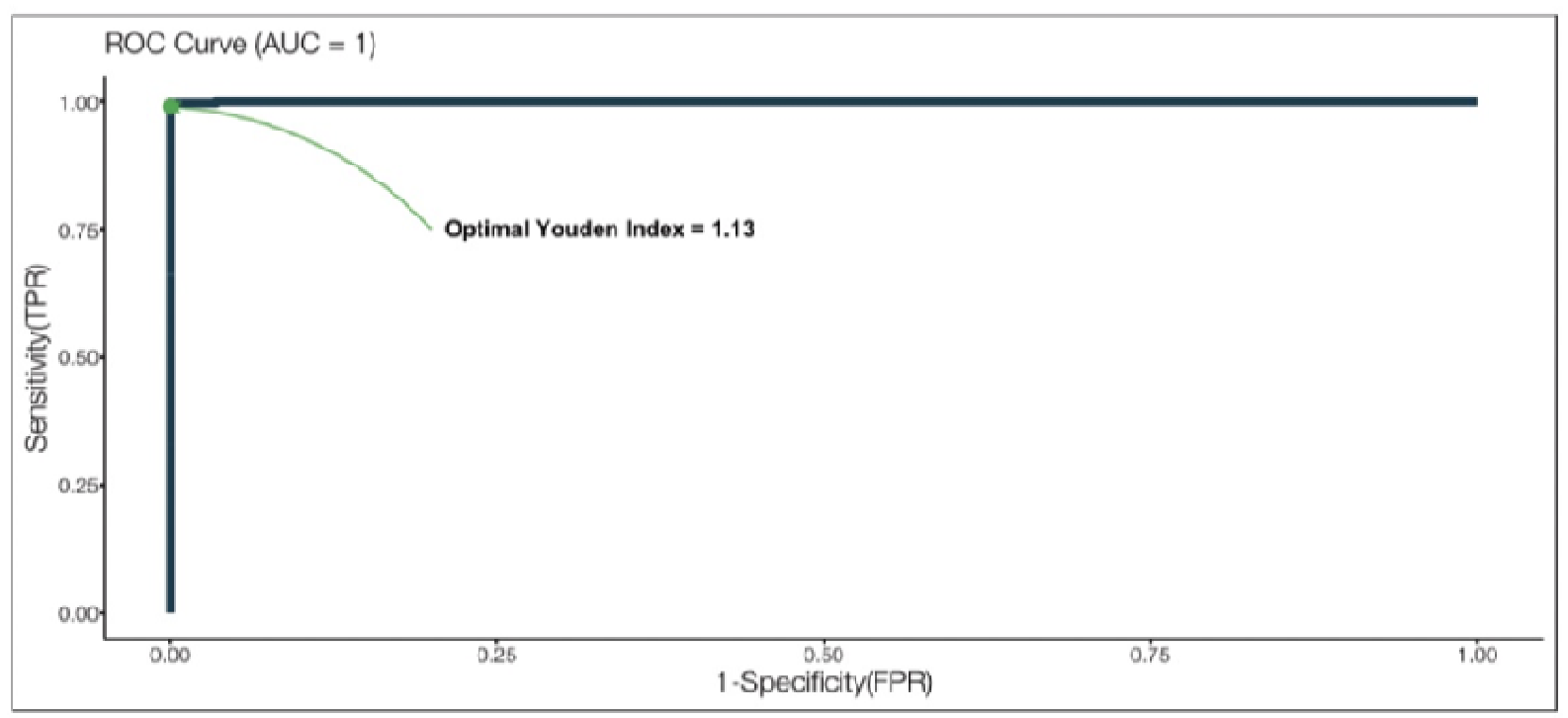

\section{Extended Data Fig. 6 | Determination of RT-LAMP threshold with a ROC curve.}

Thresholds for LAMP quality analysis were derived to determine which samples had amplified sufficiently. The exact score value for this qualitative QC metric was determined using a ROC analysis. 


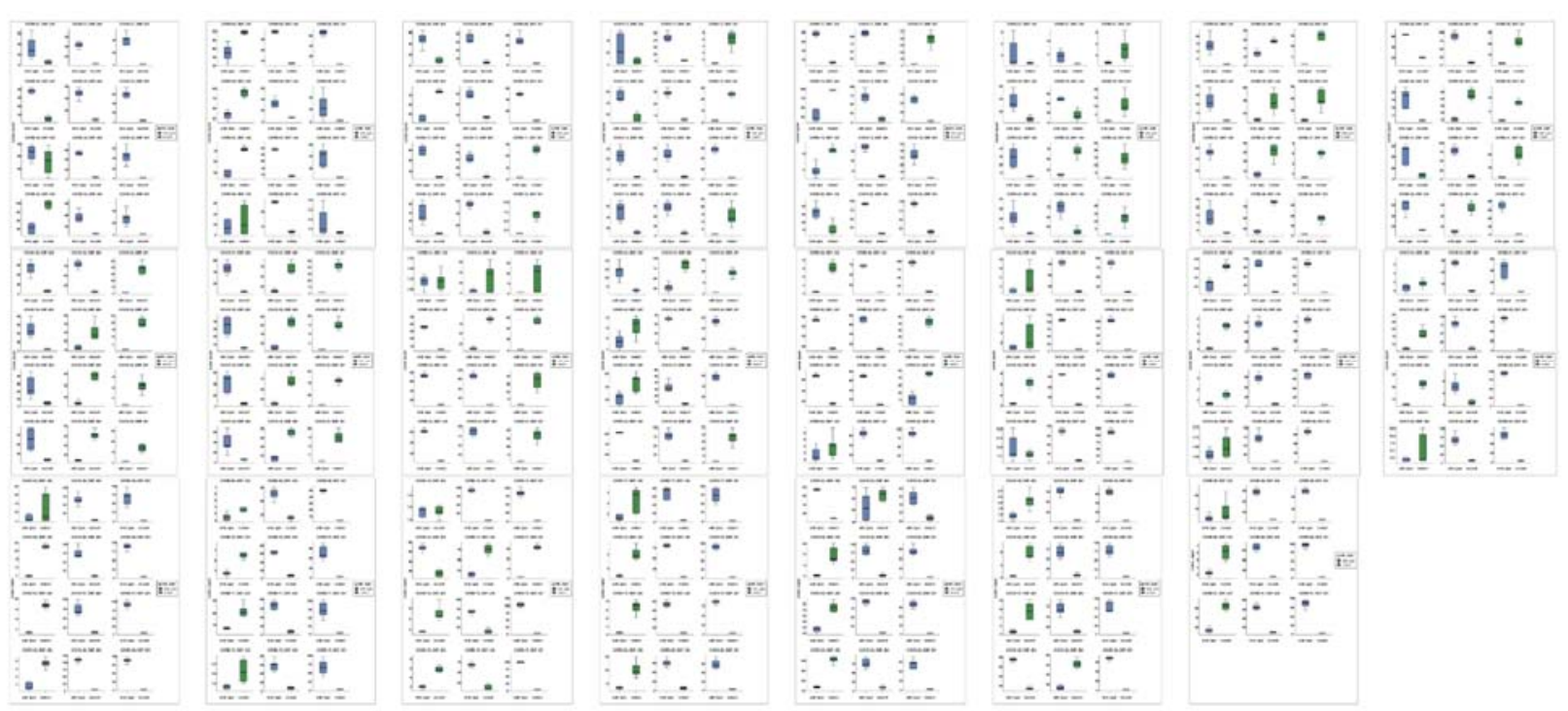

\section{Extended Data Fig. 7 | Visualization of SNP calls by the DETECTR ${ }^{\circledR}$ data analysis}

pipeline. Box plots of all the clinical samples illustrate the spread of the scaled signals for each of the samples across the replicates in the experiment. SNP calls were made on each sample agreement with the median values depicted on the box plot of the sample, which also provided an analytical confirmation of the DETECTR ${ }^{\circledR}$ results. 


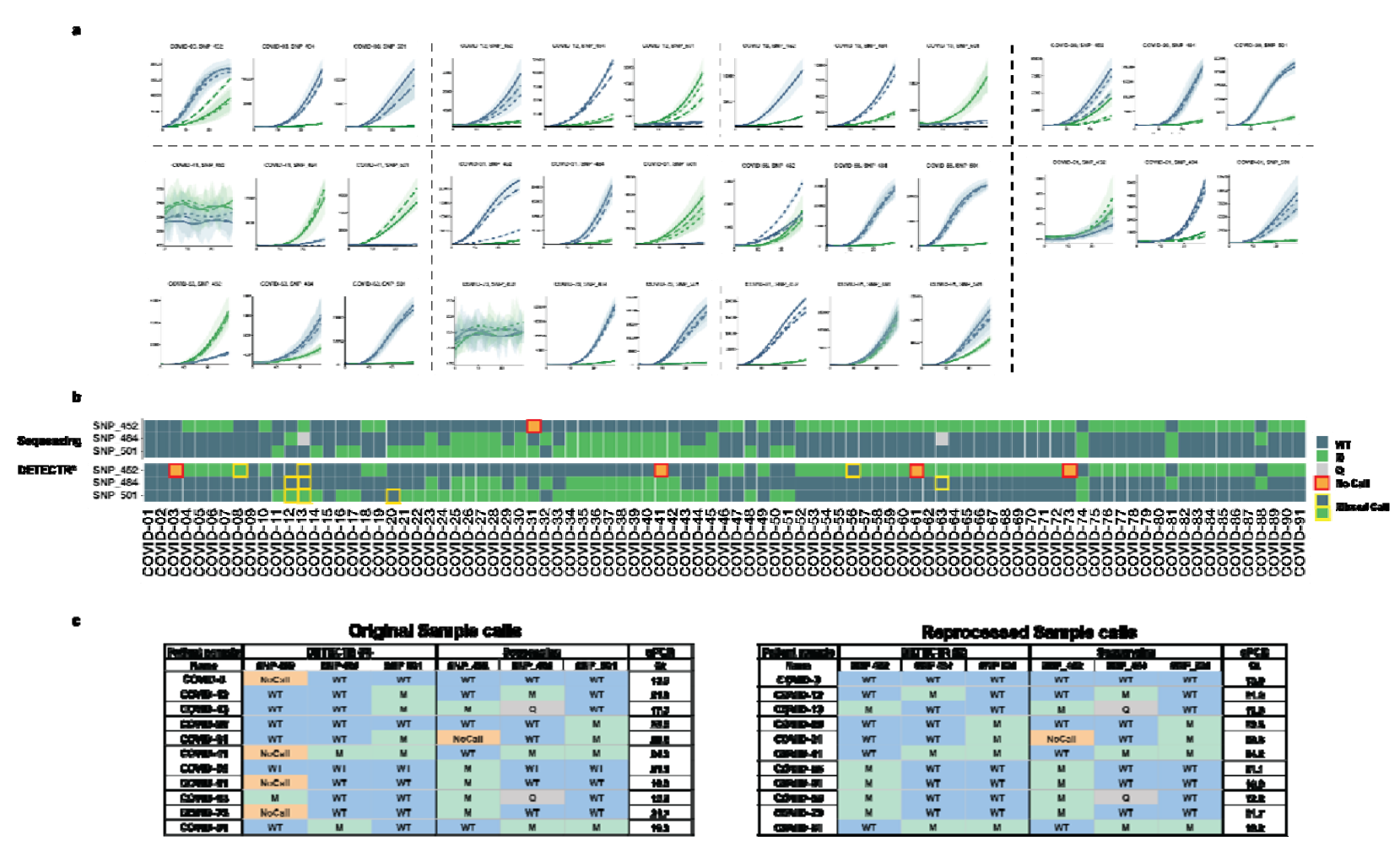

Extended Data Fig. 8 | Clinical evaluation results with clinical samples of

uncertain integrity. a, Raw fluorescence CasDx1 curves for the clinical samples with discordant DETECTR ${ }^{\circledR}$ and WGS results. WT detection is represented by blue lines and MUT detection is represented by green lines. Each line is representative of the median $\pm 1.0 \mathrm{SD}$ of the CasDx1 replicates $(n=3)$ for each guide for each of the LAMP replicates $(n=3)$, and each RT-LAMP replicate is represented by different patterned lines. $\mathbf{b}$, Visualization of the COVID Variant DETECTR ${ }^{\circledR}$ and SARS-CoV-2 WGS assays showing the alignment of final calls. Across all of the clinical samples in this cohort, 80 out of the 91 clinical sample COVID Variant DETECTR ${ }^{\circledR}$ assay calls were consistent with the SARS-CoV-2 WGS calls. c, Summary of re-testing of discordant samples from the original clinical sample shows nearly all SNP discrepancies are resolved. 


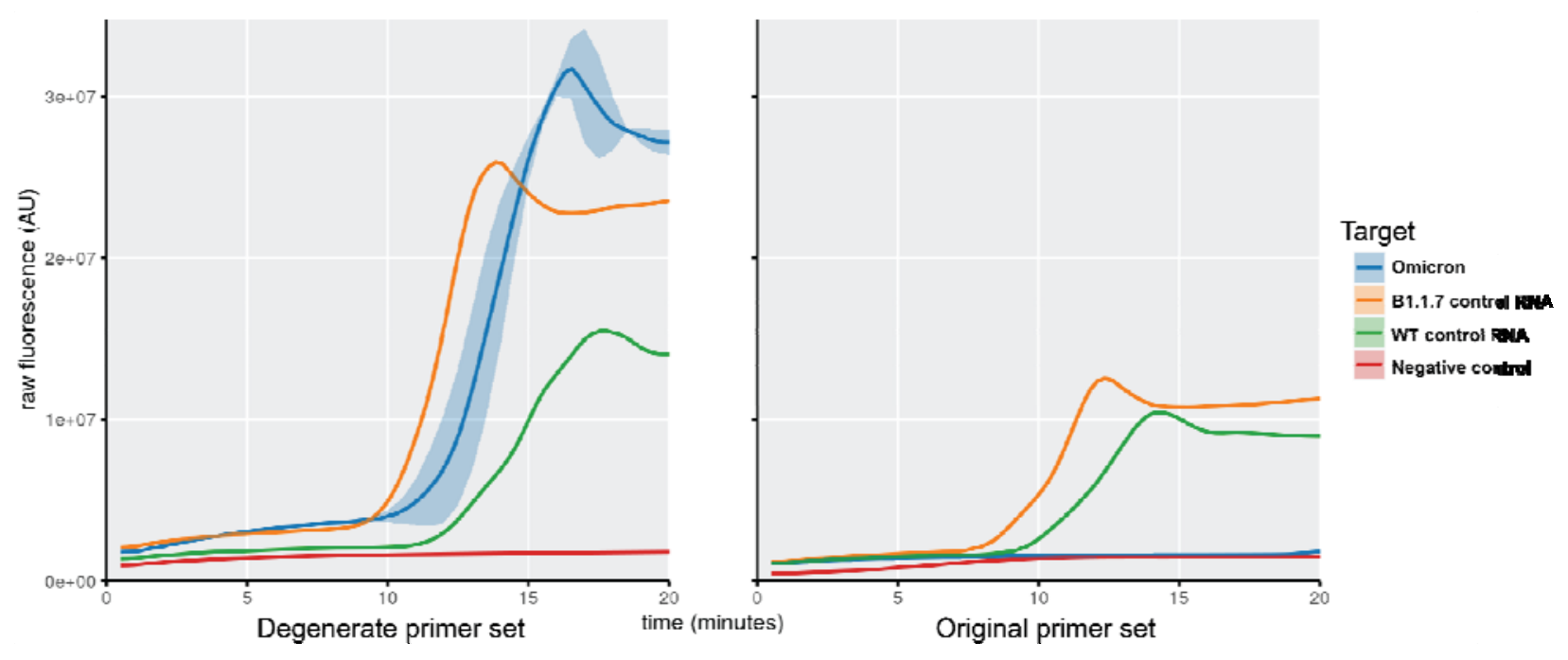

Extended Data Fig. 9 | Comparison of RT-LAMP primers for processing Omicron

clinical samples. Omicron clinical samples (blue), WT (green) control RNA and Alpha

(orange) control RNAs were amplified using the original RT-LAMP primer set and degenerate RT-LAMP primer set. The degenerate primers amplified both the controls (WT and Alpha) and Omicron samples, whereas the original primer set only amplified the control samples (WT and Alpha). 
medRxiv preprint doi: https://doi.org/10.1101/2021.11.29.21267041; this version posted February $3,2022$. The copyright holder for this preprint

(which was not certified by peer review) is the author/funder, who has granted medRxiv a license to display the preprint in perpetuity.

It is made available under a CC-BY-NC-ND 4.0 International license.

a

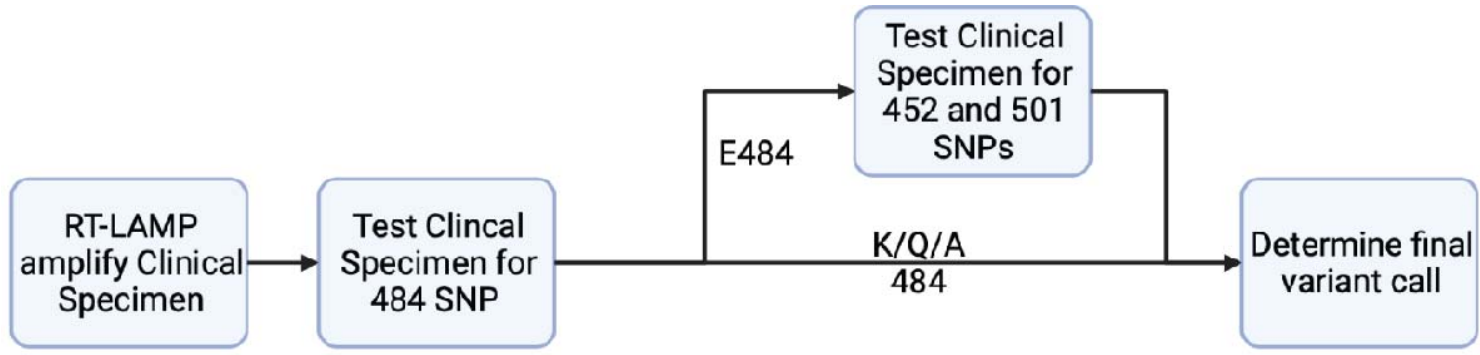

b

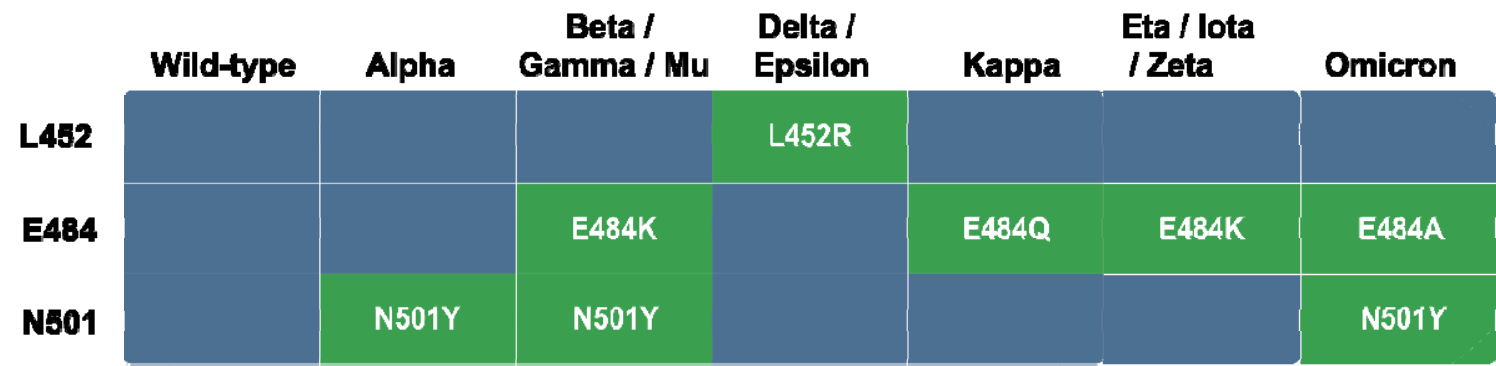

c

\begin{tabular}{|c|c|c|c|c|c|c|c|c|c|c|}
\hline Patient sample & \multicolumn{4}{|c|}{ DETECTR (R) } & \multicolumn{5}{|c|}{ Whole Genome Sequencing } & \multirow{2}{*}{$\frac{\mathrm{qPCR}}{\mathrm{Ct}}$} \\
\hline COVID-NAME & SNP_452 & SNP_484 & SNP_501 & Lineage Call & SNP_452 & SNP_484 & SNP_501 & Pango Lineage & Lineage Call & \\
\hline COVID-92 & WT & WT & WT & WT & WT & WT & WT & B.1.375 & WT & 15.3 \\
\hline COVID-93 & M & WT & WT & Delta & M & WT & WT & B.1.427 & Epsilon & 19.1 \\
\hline COVID-94 & WT & WT & WT & WT & WT & WT & WT & B.1.243 & WT & 17.9 \\
\hline COVID-95 & WT & WT & M & Alpha & WT & WT & M & B.1.1.7 & Alpha & 33.2 \\
\hline COVID-96 & WT & WT & WT & WT & WT & WT & WT & B.1.526.2 & WT & 11.4 \\
\hline COVID-97 & N/A & A & N/A & Omicron & WT & A & M & B.1.1.529 & Omicron & 16.7 \\
\hline COVID-98 & M & WT & WT & Delta & $M$ & WT & WT & B.1.617.2 & Delta & 13.9 \\
\hline COVID-99 & N/A & A & N/A & Omicron & WT & A & WT & B.1.1.529 & Omicron & 19.4 \\
\hline COVID-100 & N/A & A & N/A & Omicron & WT & A & WT & B.1.1.529 & Omicron & 14.5 \\
\hline COVID-101 & N/A & A & N/A & Omicron & WT & A & WT & B.1.1.529 & Omicron & 19.2 \\
\hline COVID-102 & N/A & Q & N/A & Kappa & M & $\mathbf{Q}$ & WT & B.1.617.1 & Kappa & 17.3 \\
\hline COVID-103 & NoCall & WT & M & Alpha & WT & WT & m & B.1.1.7 & Alpha & 23.5 \\
\hline COVID-104 & NoCall & WT & M & Alpha & NoCall & WT & M & B.1.1.7 & Alpha & 23.6 \\
\hline COVID-105 & N/A & $\kappa$ & N/A & Gamma/Eta/lota/Mu & WT & $\mathbf{k}$ & M & P.1 & Gamma & 24.2 \\
\hline COVID-106 & M & WT & WT & Delta & M & WT & WT & B.1.617.2 & Delta & 21.1 \\
\hline COVID-107 & M & WT & WT & Delta & M & WT & WT & B.1.617.2 & Delta & 16.9 \\
\hline COVID-108 & N/A & $\mathbf{Q}$ & N/A & Kappa & $M$ & $\mathbf{Q}$ & WT & B.1.617.2 & Delta & 12.2 \\
\hline COVID-109 & NoCall & WT & WT & Delta or WT & $M$ & WT & WT & B.1.617.2 & Delta & 21.7 \\
\hline COVID-110 & N/A & K & N/A & Gamma/Eta/lota/Mu & WT & $\mathbf{K}$ & M & B.1.621 & Mu & 19.2 \\
\hline COVID-111 & N/A & A & N/A & Omicron & WT & A & WT & B.1.1.529 & Omicren & 18.4 \\
\hline Covid.112 & N/A & NoCall & N/A & COVID neg & wT & A & WT & B.1.1.529 & Omicron & 27.8 \\
\hline COVID-113 & N/A & A & N/A & Omicron & WT & A & WT & B.1.1.529 & Omicron & 15.3 \\
\hline COVID-114 & N/A & A & N/A & Omicron & wT & $\Delta$ & WT & B.1.1.529 & Omicron & 12.0 \\
\hline COVID-115 & N/A & A & N/A & Omicron & WT & A & WT & B.1.1.529 & Omicron & 22.9 \\
\hline COVID-116 & N/A & A & N/A & Omicron & WT & A & WT & B.1.1.529 & Omicron & 26.4 \\
\hline COVID-117 & N/A & A & N/A & Omicron & WT & A & WT & B.1.1.529 & Omicron & 16.9 \\
\hline COVID-118 & N/A & A & N/A & Omicron & WT & A & WT & B.1.1.529 & Omicron & 22.3 \\
\hline COVID.119 & N/A & к & N/A & Gamma/Eta/Inta/MII & wT & $\mathbf{k}$ & $\mathbf{m}$ & B.1.621 & Mul & 16.7 \\
\hline COVID-120 & WT & WT & M & Alpha & WT & WT & M & B.1.1.7 & Alpha & 17.8 \\
\hline COVID-121 & M & WT & WT & Delta & $M$ & WT & WT & B.1.617.2 & Delta & 15.4 \\
\hline COVID-122 & N/A & K & N/A & Gamma/Eta/lota/Mu & M & WT & WT & B.1.617.2 & Delta & 24.2 \\
\hline COVID-123 & WT & WT & M & Alpha & WT & WT & $\mathbf{M}$ & B.1.1.7 & Alpha & 17.2 \\
\hline COVID-124 & WT & wT & M & Alpha & WI & WT & m & B.1.1.7 & Alphá & 21.2 \\
\hline COVID-125 & M & WT & WT & Delta & $\mathbf{M}$ & WT & WT & B.1.617.2 & Delta & 20.0 \\
\hline COVID-126 & M & WT & WT & Delta & M & WT & WT & B.1.617.2 & Delta & 17.3 \\
\hline COVID-127 & M & WT & WT & Delta & M & WT & WT & B.1.617.2 & Delta & 22.6 \\
\hline
\end{tabular}

d

\begin{tabular}{|c|c|c|c|c|c|c|c|c|c|}
\hline Patlent sample & \multicolumn{3}{|c|}{ DETECTR } & \multicolumn{4}{c|}{ Whole Genome Sequenclng } \\
\hline COVID-NAME & SNP_452 & SNP_484 & SNP_501 & Lineage Call & SNP_452 & SNP_484 & SNP_501 & Pango Lineage & Lineage Call \\
\hline COVID-103 & NoCall & WT & M & Alpha & WT & WT & M & B.1.1.7 & Alpha \\
\hline COVID-108 & M & Q & WT & Delta & UNK & Q & WT & B.1.617.2 & Delta \\
\hline COVID-109 & NoCall & WT & WT & Delta or WT & UNK & WT & WT & B.1.617.2 & Delta \\
\hline COVID-112 & NoCall & A & M & Omicron & UNK & UNK & UNK & B.1.1.529 & Omicron \\
\hline COVID-122 & NoCall & NoCall & NoCall & NoCall & UNK & WT & WT & B.1.617.2 & Delta \\
\hline
\end{tabular}




\section{Extended Data Fig. 10 | Results summary of final SNP calls by the COVID Variant}

DETECTR $^{\circledR}$ and WGS assays. a, Schematic of the workflow for determining the final variant calls. If the result was an A484, K484 or Q484 the final variant call was made. If the result was an E484, the sample was reflexed to DETECTR ${ }^{\circledR}$ analysis at the 452 and 501 positions to make the variant determination. $\mathbf{b}$, Interpretation table including the specific 484 SNPs. c, A summary table of the final SNP calls from the DETECTR ${ }^{\circledR}$ assay and the SARS-CoV-2 whole genome sequencing assay including the lineage classification from DETECTR ${ }^{\circledR}$ calls as well as the PANGO lineage and WHO labels assigned to the WGS calls. Ct values were obtained from the FDA EUA authorized S TaqpathTM COVID-19 RT-PCR kit. d, A summary table of the five discordant samples from the DETECTR ${ }^{\circledR}$ assay and WGS after retesting. (NoCall = lack of data generated, $\mathrm{N} / \mathrm{A}=$ assay not run) 
medRxiv preprint doi: https://doi.org/10.1101/2021.11.29.21267041; this version posted February 3, 2022. The copyright holder for this preprint

(which was not certified by peer review) is the author/funder, who has granted medRxiv a license to display the preprint in perpetuity.

It is made available under a CC-BY-NC-ND 4.0 International license .

\begin{tabular}{|c|c|c|c|c|c|}
\hline $\begin{array}{l}\text { Patient } \\
\text { sample }\end{array}$ & & & DETECTR & & \\
\hline Name & SNP 452 & SNP 484 & SNP 501 & $\begin{array}{c}\text { Generic Lineage } \\
\text { Classification }\end{array}$ & SNP 452 \\
\hline COVID-01 & WT & WT & WT & SARS-CoV-2 & WT \\
\hline COVID-02 & WT & WT & WT & SARS-CoV-2 & WT \\
\hline * COVID-03 & WT & WT & WT & SARS-CoV-2 & WT \\
\hline COVID-04 & $\mathbf{M}$ & WT & WT & Delta/Epsilon/Kappa & M \\
\hline COVID-05 & M & WT & WT & Delta/Epsilon/Kappa & M \\
\hline COVID-06 & M & WT & WT & Delta/Epsilon/Kappa & M \\
\hline COVID-07 & $\mathbf{M}$ & WT & WT & Delta/Epsilon/Kappa & M \\
\hline COVID-08 & WT & WT & WT & SARS-CoV-2 & WT \\
\hline COVID-09 & WT & WT & WT & SARS-CoV-2 & WT \\
\hline COVID-10 & M & WT & WT & Delta/Epsilon/Kappa & M \\
\hline COVID-11 & WT & WT & $\mathbf{M}$ & Alpha & WT \\
\hline$=$ COVID-12 & WT & M & WT & Eta/lota/Zeta & WT \\
\hline * COVID-13 & M & WT & WT & Delta/Epsilon/Kappa & M \\
\hline COVID-14 & WT & WT & M & Alpha & WT \\
\hline COVID-15 & WT & WT & WT & SARS-CoV-2 & WT \\
\hline COVID-16 & WT & WT & $\mathbf{M}$ & Alpha & WT \\
\hline COVID-17 & WT & WT & M & Alpha & WT \\
\hline COVID-18 & M & WT & WT & Delta/Epsilon/Kappa & M \\
\hline COVID-19 & M & WT & WT & Delta/Epsilon/Kappa & M \\
\hline${ }^{*}$ COVID-20 & WT & WT & M & Alpha & WT \\
\hline COVID-21 & WT & WT & M & Alpha & WT \\
\hline COVID-22 & WT & WT & M & Alpha & WT \\
\hline COVID-23 & WT & M & M & Beta/Gamma/Mu & WT \\
\hline COVID-24 & WT & WT & M & Alpha & WT \\
\hline COVID-25 & WT & M & M & Beta/Gamma/Mu & WT \\
\hline COVID-26 & WT & M & M & Beta/Gamma/Mu & WT \\
\hline COVID-27 & WT & M & M & Beta/Gamma/Mu & WT \\
\hline COVID-28 & WT & M & M & Beta/Gamma/Mu & WT \\
\hline COVID-29 & WT & WT & M & Alpha & WT \\
\hline COVID-30 & WT & M & M & Beta/Gamma/Mu & WT \\
\hline${ }^{*}$ COVID-31 & WT & WT & M & Alpha & NoCall \\
\hline COVID-32 & WT & M & WT & Eta/lota/Zeta & WT \\
\hline COVID-33 & WT & WT & M & Alpha & WT \\
\hline COVID-34 & WT & M & M & Beta/Gamma/Mu & WT \\
\hline COVID-35 & WT & M & M & Beta/Gamma/Mu & WT \\
\hline COVID-36 & WT & M & M & Beta/Gamma/Mu & WT \\
\hline COVID-37 & WT & M & M & Beta/Gamma/Mu & WT \\
\hline COVID-38 & WT & M & M & Beta/Gamma/Mu & WT \\
\hline COVID-39 & WT & M & M & Beta/Gamma/Mu & WT \\
\hline COVID-40 & WT & M & M & Beta/Gamma/Mu & WT \\
\hline * COVID-41 & WT & M & M & Beta/Gamma/Mu & WT \\
\hline COVID-42 & WT & M & M & Beta/Gamma/Mu & WT \\
\hline COVID-43 & WT & WT & M & Alpha & WT \\
\hline COVID-44 & WT & WT & M & Alpha & WT \\
\hline COVID-45 & WT & M & M & Beta/Gamma/Mu & WT \\
\hline COVID-46 & M & WT & WT & Delta/Epsilon/Kappa & M \\
\hline COVID-47 & M & WT & WT & Delta/Epsilon/Kappa & M \\
\hline COVID-48 & WT & WT & M & Alpha & WT \\
\hline COVID-49 & M & WT & WT & Delta/Epsilon/Kappa & M \\
\hline COVID-50 & WT & WT & M & Alpha & WT \\
\hline COVID-51 & WT & WT & M & Alpha & WT \\
\hline COVID-52 & M & WT & WT & Delta/Epsilon/Kappa & M \\
\hline COVID-53 & M & WT & WT & Delta/Epsilon/Kappa & M \\
\hline COVID-54 & M & WT & WT & Delta/Epsilon/Kappa & M \\
\hline COVID-55 & M & WT & WT & Delta/Epsilon/Kappa & M \\
\hline${ }^{*}$ COVID-56 & M & WT & WT & Delta/Epsilon/Kappa & M \\
\hline COVID-57 & M & WT & WT & Delta/Epsilon/Kappa & M \\
\hline COVID-58 & M & WT & WT & Delta/Epsilon/Kappa & M \\
\hline COVID-59 & M & WT & WT & Delta/Epsilon/Kappa & M \\
\hline COVID-60 & M & WT & WT & Delta/Epsilon/Kappa & M \\
\hline * COVID-61 & $\mathbf{M}$ & WT & WT & Delta/Epsilon/Kappa & M \\
\hline COVID-62 & M & WT & WT & Delta/Epsilon/Kappa & M \\
\hline${ }^{*}$ COVID-63 & M & WT & WT & Delta/Epsilon/Kappa & M \\
\hline COVID-64 & M & WT & WT & Delta/Epsilon/Kappa & M \\
\hline COVID-65 & M & WT & WT & Delta/Epsilon/Kappa & M \\
\hline COVID-66 & M & WT & WT & Delta/Epsilon/Kappa & M \\
\hline COVID-67 & M & WT & WT & Delta/Epsilon/Kappa & M \\
\hline COVID-68 & M & WT & WT & Delta/Epsilon/Kappa & M \\
\hline COVID-69 & M & WT & WT & Delta/Epsilon/Kappa & M \\
\hline COVID-70 & M & WT & WT & Delta/Epsilon/Kappa & M \\
\hline COVID-71 & $\mathbf{M}$ & WT & WT & Delta/Epsilon/Kappa & M \\
\hline COVID-72 & $\mathbf{M}$ & WT & WT & Delta/Epsilon/Kappa & M \\
\hline * COVID-73 & M & WT & WT & Delta/Epsilon/Kappa & M \\
\hline COVID-74 & WT & M & M & Beta/Gamma/Mu & WT \\
\hline COVID-75 & M & WT & WT & Delta/Epsilon/Kappa & M \\
\hline COVID-76 & $\mathbf{M}$ & WT & WT & Delta/Epsilon/Kappa & M \\
\hline COVID-77 & M & WT & WT & Delta/Epsilon/Kappa & M \\
\hline COVID-78 & M & WT & WT & Delta/Epsilon/Kappa & M \\
\hline COVID-79 & M & WT & WT & Delta/Epsilon/Kappa & M \\
\hline COVID-80 & M & WT & WT & Delta/Epsilon/Kappa & M \\
\hline${ }^{*}$ COVID-81 & WT & M & M & Beta/Gamma/Mu & WT \\
\hline COVID-82 & $\mathbf{M}$ & WT & WT & Delta/Epsilon/Kappa & M \\
\hline COVID-83 & M & WT & WT & Delta/Epsilon/Kappa & M \\
\hline COVID-84 & $\mathbf{M}$ & WT & WT & Delta/Epsilon/Kappa & M \\
\hline COVID-85 & $\mathbf{M}$ & WT & WT & Delta/Epsilon/Kappa & M \\
\hline COVID-86 & M & WT & WT & Delta/Epsilon/Kappa & M \\
\hline COVID-87 & M & WT & WT & Delta/Epsilon/Kappa & M \\
\hline COVID-88 & WT & M & WT & Eta/lota/Zeta & WT \\
\hline COVID-89 & M & WT & WT & Delta/Epsilon/Kappa & M \\
\hline COVID-90 & M & WT & WT & Delta/Epsilon/Kappa & M \\
\hline COVID-91 & M & WT & WT & Delta/Epsilon/Kappa & M \\
\hline
\end{tabular}


medRxiv preprint doi: https://doi.org/10.1101/2021.11.29.21267041; this version posted February 3, 2022. The copyright holder for this preprint (which was not certified by peer review) is the author/funder, who has granted medRxiv a license to display the preprint in perpetuity.

It is made available under a CC-BY-NC-ND 4.0 International license .

\section{Supplementary Table 1 | Overall results summary of final SNP calls by the}

DETECTR $^{\circledR}$ assay and viral WGS. A summary table of the final SNP calls from the DETECTR $^{\circledR}$ assay and the SARS-CoV-2 whole genome sequencing assay after discordant testing. The table includes the lineage classification from DETECTR ${ }^{\circledR}$ calls as well as the PANGO lineage and WHO labels assigned to the WGS calls. Ct values from running an FDA EUA authorized SARS-CoV-2 RT-PCR assay, the Taqpath ${ }^{\mathrm{TM}}$ COVID-19 RT-PCR kit, are shown. Discordant samples were reflexed back for reprocessing (*); COVID-63 was classified as a Delta variant by WGS despite its Q484 SNP call. (†).

\begin{tabular}{|c|c|c|c|c|c|c|c|c|c|c|}
\hline \multirow{2}{*}{$\begin{array}{l}\text { Patient specimen } \\
\text { Sample Name }\end{array}$} & \multicolumn{4}{|c|}{ DETECTR (R) } & \multicolumn{5}{|c|}{ Whole Genome Sequencing } & \multirow{2}{*}{$\frac{\mathrm{qPCR}}{\mathrm{Ct}}$} \\
\hline & SNP_452 & SNP_484 & SNP_501 & Lineage Call & SNP_452 & SNP_484 & SNP_501 & Pango Lineage & Lineage/Variant Call & \\
\hline Sample-1 & N/A & NoCall & N/A & COVID ND & NoCall & NoCall & NoCall & COVID neg & COVID ND & N/A \\
\hline Sample-2 & N/A & NoCall & N/A & COVID ND & NoCall & NoCall & NoCall & COVID neg & COVID ND & N/A \\
\hline Sample-3 & N/A & NoCall & N/A & COVID ND & NoCall & NoCall & NoCall & $>30 \mathrm{Ct}$ & COVID ND & 38.6 \\
\hline Sample-4 & N/A & NoCall & N/A & COVID ND & NoCall & NoCall & NoCall & $>30 \mathrm{Ct}$ & COVID ND & 33.2 \\
\hline Sample-5 & N/A & NoCall & N/A & COVID ND & NoCall & NoCall & NoCall & COVID neg & COVID ND & N/A \\
\hline Sample-6 & N/A & NoCall & N/A & COVID ND & NoCall & NoCall & NoCall & $>30 \mathrm{Ct}$ & COVID ND & 38.7 \\
\hline Sample-7 & N/A & NoCall & N/A & COVID ND & NoCall & NoCall & NoCall & $>30 \mathrm{Ct}$ & COVID ND & 35.8 \\
\hline Sample-8 & N/A & NoCall & N/A & COVID ND & NoCall & NoCall & NoCall & $>30 \mathrm{Ct}$ & COVID ND & 32.1 \\
\hline Sample-9 & N/A & NoCall & N/A & COVID ND & NoCall & NoCall & NoCall & $>30 \mathrm{Ct}$ & COVID ND & 30.1 \\
\hline Sample-10 & N/A & NoCall & N/A & COVID ND & NoCall & NoCall & NoCall & $>30 \mathrm{Ct}$ & COVID ND & 36.9 \\
\hline Sample-11 & N/A & NoCall & N/A & COVID ND & NoCall & NoCall & NoCall & $>30 \mathrm{Ct}$ & COVID ND & 34.7 \\
\hline Sample-12 & N/A & NoCall & N/A & COVID ND & NoCall & NoCall & NoCall & Covid neg & COVID ND & N/A \\
\hline
\end{tabular}

\section{Supplementary Table 2 | Summary of samples showing no COVID detection. A}

summary table of the clinical specimens with no signal in the RT-LAMP nor the DETECTR ${ }^{\circledR}$ reactions. These samples were called "COVID Not Detected (ND)" by both the COVID-19 variant DETECTR ${ }^{\circledR}$ assay and the SARS-CoV-2 whole genome sequencing assays. Ct values were obtained from the FDA EUA authorized S Taqpath $^{\text {TM }}$ COVID-19 RT-PCR kit. (NoCall = lack of data generated, N/A = assay not run). 


\begin{tabular}{|c|c|c|c|}
\hline Type & Name & Sequence & Source \\
\hline \multirow{15}{*}{ 咅 } & Drt_l492 & 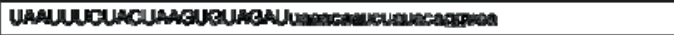 & Dhemmaxon ar Syntheys \\
\hline & DXI_Amer & 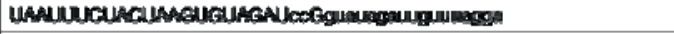 & Dhemacson of Synthegs \\
\hline & Dar1_EA94 & 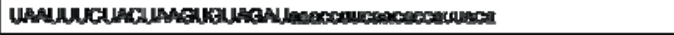 & Dhemecon of Symthepo \\
\hline & DxIJKASA & 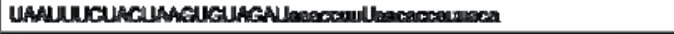 & Dhemacon or Symthege \\
\hline & DxI_NEOH & 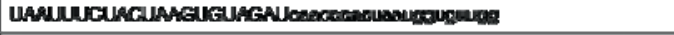 & Dhemecon of Syrithego \\
\hline & DXLYVor & 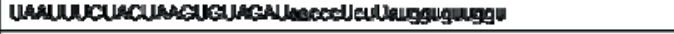 & Dhemacen of Pyntheges \\
\hline & Dri_Jaen va & 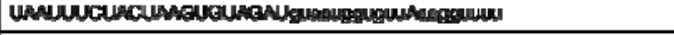 & Smathege \\
\hline & Dxt_a494 & 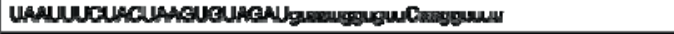 & Sonthesp \\
\hline & Dori, Aman & 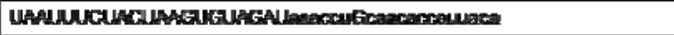 & Shathespe \\
\hline & 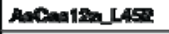 & 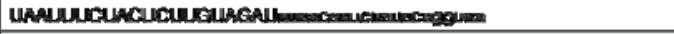 & Dhemuacon of Simthego \\
\hline & AsCan12aAas & 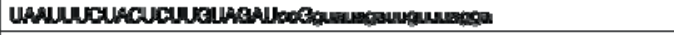 & Dhamason or 8 mithese \\
\hline & AECASIZ_EEAS & 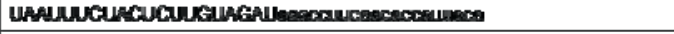 & Dhemeeron or Symthese \\
\hline & Ancas 12a, KNA & 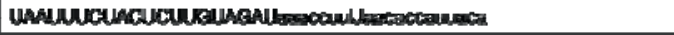 & Dhemberon ar Byntheres \\
\hline & Anclan12a_Msor & 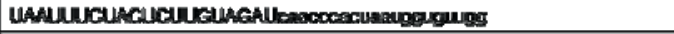 & Dhemacon a Synthego \\
\hline & Aschatean_rsor & 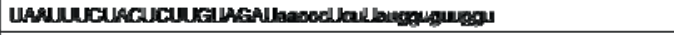 & Dhemason or Dimthege \\
\hline 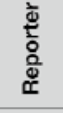 & ssoma reportar & 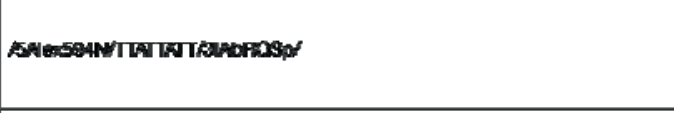 & DT \\
\hline \multirow{4}{*}{ 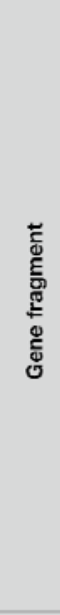 } & 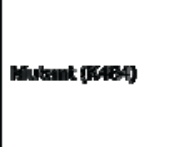 & 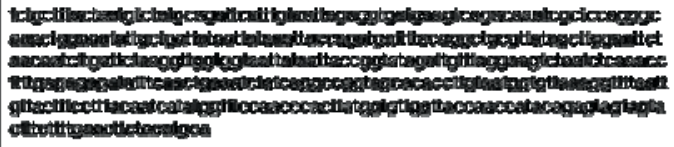 & Theta Blosedentes: \\
\hline & Wild Type & 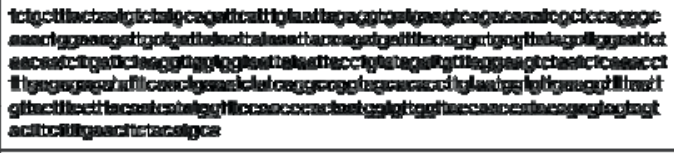 & Theild Bicecionose \\
\hline & 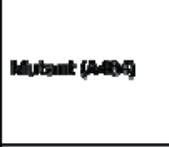 & 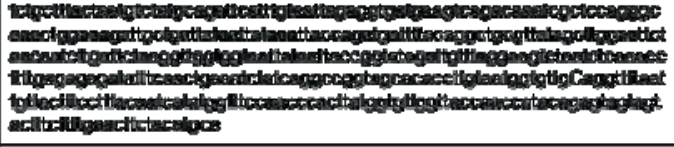 & Thlok Blociences \\
\hline & Intumen & 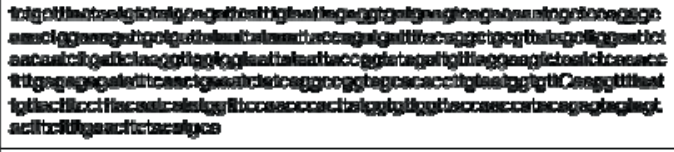 & 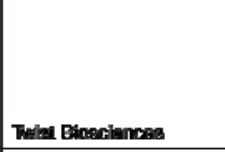 \\
\hline \multirow{12}{*}{ 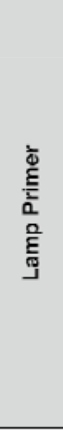 } & $\operatorname{sent}-\mathrm{Fa}$ & 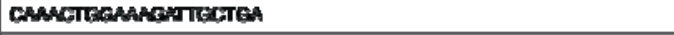 & Ewoins eanticics \\
\hline & setr-15s & TAGTACTACTCTERAIEGTS & Errothe Centanks \\
\hline & ment-EAP & 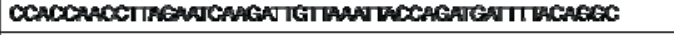 & Ewrofins Genemics \\
\hline & sett-Fip & 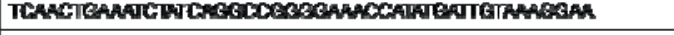 & Emotha Canemirs \\
\hline & att-L & 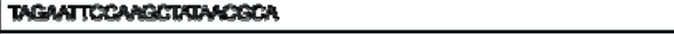 & Emoins Gantenive \\
\hline & sent-LB & TALCACNCCTTETANGGTO & Encths: Benomiss \\
\hline & $\operatorname{set} 2 \mathrm{~F}$ & 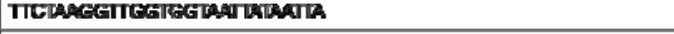 & Erotha Gensonka \\
\hline & atas & CATTEMLGTTGAAMTTACACAT & Eurothe Caneniks \\
\hline & natar & 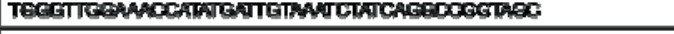 & Emoins Gancenibs \\
\hline & matip & 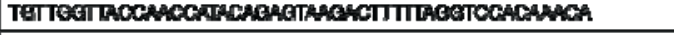 & Earciths Sensenks \\
\hline & $\operatorname{set} \mathbf{L} \mathbf{F}$ & 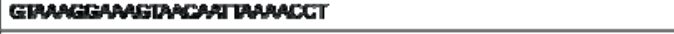 & Ewoins Cenomics \\
\hline & state & AACTTCTACNTOCACCACCAR & Evolina Conombe \\
\hline \multirow{12}{*}{ 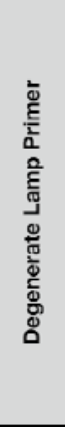 } & sett-Fus & CaAnctseanaATTECTEA & Eusithe Genomits \\
\hline & $\operatorname{sect-13}$ & TACTACTACTCTELATGGTS & Errothe Cenemles \\
\hline & ant-map & 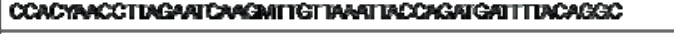 & Ewolhm Ganomits \\
\hline & ant-F" & 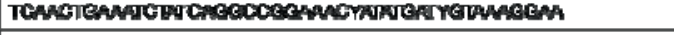 & Emotine Gentembs \\
\hline & sott-LF & 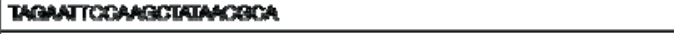 & Ereiths Ganemize \\
\hline & $\operatorname{sot} t-1 \mathrm{~B}$ & TARAMMCETGTINATESTG & Emoing Cenemits \\
\hline & seters & 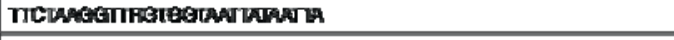 & Ewoths Canemites \\
\hline & sotat & 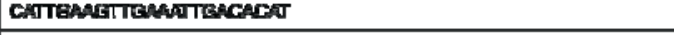 & Evefine Genemise \\
\hline & $\operatorname{set}-\operatorname{mip}$ & 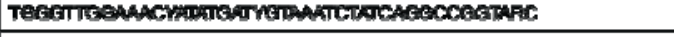 & Emoins Sanemibs \\
\hline & $\operatorname{sen} 2-F P$ & TETTEGTVACCANCCANACAGADACACTIITLACGTCCACAAACA & Eworins canomics \\
\hline & $\sec 15$ & 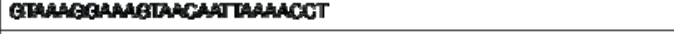 & Emothe Gancentas \\
\hline & $\operatorname{mat} \mathbf{L}$ & АACTTCTACATCCACCACCAA & Emoins Conemics \\
\hline
\end{tabular}


medRxiv preprint doi: https://doi.org/10.1101/2021.11.29.21267041; this version posted February 3, 2022. The copyright holder for this preprint (which was not certified by peer review) is the author/funder, who has granted medRxiv a license to display the preprint in perpetuity. It is made available under a CC-BY-NC-ND 4.0 International license .

Supplementary Table 3 | Nucleic acid sequences used in this study. A list of guide

RNAs, reporter molecules, LAMP primers and synthetic gene fragment targets with their respective suppliers. ( ${ }^{*}$ indicates LAMP primers that contain degenerate nucleotides)

\section{REFERENCES}

1. Otto, S. P. et al. The origins and potential future of SARS-CoV-2 variants of concern in the evolving COVID-19 pandemic. Curr Biol 31, R918-R929 (2021).

2. Walensky, R. P., Walke, H. T. \& Fauci, A. S. SARS-CoV-2 Variants of Concern in the United States-Challenges and Opportunities. Jama 325, 1037-1038 (2021).

3. Deng, X. et al. Genomic surveillance reveals multiple introductions of SARSCoV-2 into Northern California. Science 369, 582-587 (2020).

4. Truelove, S. et al. Projected resurgence of COVID-19 in the United States in July-December 2021 resulting from the increased transmissibility of the Delta variant and faltering vaccination. Medrxiv 2021.08.28.21262748 (2021) doi:10.1101/2021.08.28.21262748.

5. Washington, N. L. et al. Emergence and rapid transmission of SARS-CoV-2 B.1.1.7 in the United States. Cell 184, 2587-2594.e7 (2021).

6. Okereke, M. Spread of the Delta Coronavirus Variant: Africa Must Be on Watch. Public Heal Pract 2, 100209 (2021).

7. Sabino, E. C. et al. Resurgence of COVID-19 in Manaus, Brazil, despite high seroprevalence. Lancet Lond Engl 397, 452-455 (2021).

8. Servellita, V. et al. Predominance of antibody-resistant SARS-CoV-2 variants in vaccine breakthrough cases from the San Francisco Bay Area, California. Nat Microbiol 1-12 (2022) doi:10.1038/s41564-021-01041-4.

9. Kustin, T. et al. Evidence for increased breakthrough rates of SARS-CoV-2 variants of concern in BNT162b2-mRNA-vaccinated individuals. Nat Med 27, 1379-1384 (2021).

10.Jung, J., Sung, H. \& Kim, S.-H. Covid-19 Breakthrough Infections in Vaccinated Health Care Workers. New Engl J Med 385, 1629-1631 (2021).

11. Brown, C. M. et al. Outbreak of SARS-CoV-2 Infections, Including COVID-19 Vaccine Breakthrough Infections, Associated with Large Public Gatherings Barnstable County, Massachusetts, July 2021. Morbidity Mortal Wkly Rep 70, 1059-1062 (2021).

12. Campbell, F. et al. Increased transmissibility and global spread of SARS-CoV-2 variants of concern as at June 2021. Eurosurveillance 26, 2100509 (2021).

13. Harvey, W. T. et al. SARS-CoV-2 variants, spike mutations and immune escape. Nat Rev Microbiol 19, 1-16 (2021).

14. Garcia-Beltran, W. F. et al. Multiple SARS-CoV-2 variants escape neutralization by vaccine-induced humoral immunity. Cell 184, 2523-2523 (2021). 
medRxiv preprint doi: https://doi.org/10.1101/2021.11.29.21267041; this version posted February 3, 2022. The copyright holder for this preprint (which was not certified by peer review) is the author/funder, who has granted medRxiv a license to display the preprint in perpetuity. It is made available under a CC-BY-NC-ND 4.0 International license .

15. Focosi, D., Tuccori, M., Baj, A. \& Maggi, F. SARS-CoV-2 Variants: A Synopsis of In Vitro Efficacy Data of Convalescent Plasma, Currently Marketed Vaccines, and Monoclonal Antibodies. Viruses 13, 1211 (2021).

16. Munnink, B. B. O. et al. The next phase of SARS-CoV-2 surveillance: real-time molecular epidemiology. Nat Med 27, 1518-1524 (2021).

17. RockefellerFoundation. Toward a National Genomic Surveillance Network. Rockefeller Foundation https://www.rockefellerfoundation.org/wpcontent/uploads/2021/03/Toward-a-National-Genomic-Surveillance-Network1.pdf (2021).

18. Harper, H. et al. Detecting SARS-CoV-2 variants with SNP genotyping. Plos One 16, e0243185 (2021).

19. Verosloff, M. S. et al. CRISPR-Cas enzymes: The toolkit revolutionizing diagnostics. Biotechnol J 2100304 (2021) doi:10.1002/biot.202100304.

20. Broughton, J. P. et al. CRISPR-Cas12-based detection of SARS-CoV-2. Nat Biotechnol 38, 870-874 (2020).

21. Puig, H. de et al. Minimally instrumented SHERLOCK (miSHERLOCK) for CRISPR-based point-of-care diagnosis of SARS-CoV-2 and emerging variants. Sci Adv 7, eabh2944 (2021).

22. Patchsung, M. et al. Clinical validation of a Cas13-based assay for the detection of SARS-CoV-2 RNA. Nat Biomed Eng 4, 1140-1149 (2020).

23. Fozouni, P. et al. Amplification-free detection of SARS-CoV-2 with CRISPRCas13a and mobile phone microscopy. Cell 184, 323-333.e9 (2021).

24. FDA. SARS-CoV-2 RNA DETECTR Assay. https://www.fda.gov/media/139937/download (2020).

25. FDA. Sherlock ${ }^{\mathrm{TM}}$ CRISPR SARS-CoV-2 kit. https://www.fda.gov/media/137748/download (2020).

26. FDA. DETECTR BOOST SARS-CoV-2 Reagent Kit. https://www.fda.gov/media/155640/download (2022)

27. Gootenberg, J. S. et al. Multiplexed and portable nucleic acid detection platform with Cas13, Cas12a, and Csm6. Science 360, 439-444 (2018).

28. Chiu, C. Cutting-Edge Infectious Disease Diagnostics with CRISPR. Cell Host Microbe 23, 702-704 (2018).

29. Chen, J. S. et al. CRISPR-Cas12a target binding unleashes indiscriminate single-stranded DNase activity. Science 360, 436-439 (2018).

30. Zhang, L. et al. Ten emerging SARS-CoV-2 spike variants exhibit variable infectivity, animal tropism, and antibody neutralization. Commun Biology 4, 1196 (2021).

31. Corbett, K. S. et al. SARS-CoV-2 mRNA Vaccine Design Enabled by Prototype Pathogen Preparedness. Nature 586, 567-571 (2020).

32. Polack, F. P. et al. Safety and Efficacy of the BNT162b2 mRNA Covid-19 Vaccine. New Engl J Medicine 383, NEJMoa2034577 (2020).

33. Bos, R. et al. Ad26 vector-based COVID-19 vaccine encoding a prefusionstabilized SARS-CoV-2 Spike immunogen induces potent humoral and cellular immune responses. Npj Vaccines 5, 91 (2020).

34. Konings, F. et al. SARS-CoV-2 Variants of Interest and Concern naming scheme conducive for global discourse. Nat Microbiol 6, 821-823 (2021). 
medRxiv preprint doi: https://doi.org/10.1101/2021.11.29.21267041; this version posted February 3, 2022. The copyright holder for this preprint (which was not certified by peer review) is the author/funder, who has granted medRxiv a license to display the preprint in perpetuity. It is made available under a CC-BY-NC-ND 4.0 International license .

35. Malta, F. de M. et al. Mass molecular testing for COVID19 using NGS-based technology and a highly scalable workflow. Sci Rep-uk 11, 7122 (2021).

36. Igl $\square \mathrm{i}$, Z. et al. Clinical Evaluation of Roche SD Biosensor Rapid Antigen Test for SARS-CoV-2 in Municipal Health Service Testing Site, the Netherlands - Volume 27, Number 5-May 2021 - Emerging Infectious Diseases journal - CDC. Emerg Infect Dis 27, 1323-1329 (2021).

37. Broccanello, C. et al. Comparison of three PCR-based assays for SNP genotyping in plants. Plant Methods 14, 28 (2018).

38. McGuigan, F. E. A. \& Ralston, S. H. Single nucleotide polymorphism detection:allelic discrimination using TaqMan. Psychiatr Genet 12, 133-136 (2002).

39. Viana, R. et al. Rapid epidemic expansion of the SARS-CoV-2 Omicron variant in southern Africa. Nature 1-10 (2022) doi:10.1038/s41586-022-04411-y.

40. Flemming, A. Omicron, the great escape artist. Nat Rev Immunol, doi: 10.1038/s41577-022-00676-6 (2022).

41. VanBlargan, L.A., et al. An infectious SARS-CoV-2 B.1.1.529 Omicron virus escapes neutralization by therapeutic monoclonal antibodies. Nat Med, doi: 10.1038/s41591-021-01678-y (2022).

42. Hallett, S. We need increased targeted measures now to slow the spread of omicron. BMJ 375, n3133, doi: 10.1136/bmj.n3133 (2021).

43. Majumdar, S. \& Sarkar, R. Mutational and phylogenetic analyses of the two lineages of the Omicron variant. J Med Virol, doi: 10.1002/jmv.27558 (2021).

44. Mertens, J., et al. Monitoring the SARS-CoV-2 pandemic: screening algorithm with single nucleotide polymorphism detection for the rapid identification of established and emerging variants. Clin Microbiol Infect 28, 124-129, doi: 10.1016/j.cmi.2021.09.007 (2022).

45. Aleem, A., Akbar Samad, A.B. \& Slenker, A.K. Emerging Variants of SARS-CoV2 And Novel Therapeutics Against Coronavirus (COVID-19). in StatPearls (Treasure Island (FL), 2022).

46. Feder, K.A., et al. Association of E484K spike protein mutation with SARS-CoV-2 infection in vaccinated persons---Maryland, January - May 2021. Clin Infect Dis, doi: $10.1093 / \mathrm{cid} / \mathrm{ciab} 762$ (2021).

47. Team, C.C.-R. SARS-CoV-2 B.1.1.529 (Omicron) Variant - United States, December 1-8, 2021. MMWR Morb Mortal Wkly Rep 70, 1731-1734, doi: 10.15585/mmwr.mm7050e1 (2021).

48. Singh, J., Pandit, P., McArthur, A. G., Banerjee, A. \& Mossman, K. Evolutionary trajectory of SARS-CoV-2 and emerging variants. Virol J 18, 166 (2021).

49. Arizti-Sanz, J. et al. Equipment-free detection of SARS-CoV-2 and Variants of Concern using Cas13. Medrxiv 2021.11.01.21265764 (2021) doi:10.1101/2021.11.01.21265764.

50. Welch, N. L. et al. Multiplexed CRISPR-based microfluidic platform for clinical testing of respiratory viruses and SARS-CoV-2 variants. (2021) doi:10.1101/2021.12.14.21267689.

51. Cao, Y., et al. Omicron escapes the majority of existing SARS-CoV-2 neutralizing antibodies. Nature, doi: 10.1038/s41586-021-04385-3 (2021). 
52. Kibbe, W. A. OligoCalc: an online oligonucleotide properties calculator. Nucleic Acids Res 35, W43-W46 (2007).

53. Plitnick, J., et al. Whole-Genome Sequencing of SARS-CoV-2: Assessment of the Ion Torrent AmpliSeq Panel and Comparison with the Illumina MiSeq ARTIC Protocol. J Clin Microbiol 59, e0064921, doi: 10.1128/JCM.00649-21 (2021).

54. Quick, J. et al. Multiplex PCR method for MinION and Illumina sequencing of Zika and other virus genomes directly from clinical samples. Nat Protoc 12 , 1261-1276 (2017).

55. O'Toole, Á. et al. Assignment of Epidemiological Lineages in an Emerging Pandemic Using the Pangolin Tool. Virus Evol 7, veab064- (2021).

56. Rambaut, A. et al. A dynamic nomenclature proposal for SARS-CoV-2 lineages to assist genomic epidemiology. Nat Microbiol 5, 1403-1407 (2020).

57. R_Core_Team. R: A Language and Environmental for Statistical Computing. (R Core Team, 2018). 